\title{
The Unification Problem for Confluent Semi-Constructor TRSs*
}

\author{
Ichiro MITSUHASHI ${ }^{\dagger a}$, Nonmember, Michio OYAMAGUCHI ${ }^{\dagger \dagger}$, Member $^{\circ}$ \\ and Kunihiro MATSUURA ${ }^{\dagger \dagger}$, Nonmember
}

\begin{abstract}
SUMMARY The unification problem for term rewriting systems (TRSs) is the problem of deciding, for a TRS $R$ and two terms $s$ and $t$, whether $s$ and $t$ are unifiable modulo $R$. We have shown that the problem is decidable for confluent simple TRSs. Here, a simple TRS means one where the right-hand side of every rewrite rule is a ground term or a variable. In this paper, we extend this result and show that the unification problem for confluent semi-constructor TRSs is decidable. Here, a semi-constructor TRS means one where all defined symbols appearing in the right-hand side of each rewrite rule occur only in its ground subterms.

key words: term rewriting system, decision problem, unification, semiconstructor
\end{abstract}

\section{Introduction}

The unification problem for term rewriting systems (TRSs) is the problem of deciding, for a TRS $R$ and two terms $s$ and $t$, whether $s$ and $t$ are unifiable modulo $R$. This problem is undecidable in general, even if we restrict ourselves to either right-ground TRSs [13] or terminating, confluent, monadic, and linear TRSs [9]. Here, a TRS is monadic if the height of the right-hand side of every rewrite rule is at most one [15]. On the other hand, it is known that unification is decidable for shallow TRSs [2], canonical right-ground TRSs [5], semi-linear TRSs [6], linear standard TRSs [12], and confluent right-ground TRSs [14]. We have shown that the unification problem is decidable for confluent simple TRSs [9]. Here, a TRS is simple if the right-hand side of every rewrite rule is a ground term or a variable. For the class of simple TRSs which may not be confluent, it is known that the unification problem is undecidable, because unification is undecidable for non-confluent TRSs, even if we restrict ourselves to right-ground TRSs [13]. In this paper, we extend the result of [9] and show that unification for confluent semi-constructor TRSs is decidable. Here, a TRS is semi-

\footnotetext{
Manuscript received September 1, 2008.

Manuscript revised June 8, 2010.

†The author is with the Center for Information Technologies and Networks, Mie University, Tsu-shi, 514-8507 Japan.

${ }^{\dagger \dagger}$ The authors are with the Graduate School of Engineering, Mie University, Tsu-shi, 514-8507 Japan.

${ }^{*}$ This paper is an extended version of the latter half of the paper: I. Mitsuhashi, M. Oyamaguchi, Y. Ohta, and T. Yamada, "The joinability and unification problems for confluent semiconstructor TRSs," in RTA-04 Rewriting Techniques and Applications, LNCS3091, pp.285-300, 2004 (that of the first half is [10]). Since a complete proof of the latter half was not presented due to limited space, a complete one given in this paper is new.

a)E-mail: mitsuhashi@cc.mie-u.ac.jp

DOI: 10.1587/transinf.E93.D.2962
}

constructor if all defined symbols appearing in the righthand side of each rewrite rule occur only in its ground subterms. The class of semi-constructor TRSs was introduced by the authors in order to explore the border between decidable and undecidable classes of the decision problems and in particular to find nontrivial non-right-linear subclasses of TRSs which possess the decidability of unification. This class properly includes the class of simple TRSs. Thus, confluence is a necessary condition to investigate the decidability of unification for semi-constructor TRSs.

In this paper, we use a new unification algorithm obtained by refining those of [9], [14] to show the decidability of the unification problem for confluent semi-constructor TRSs. The main difference between the algorithms of the present paper and of the previous works [9], [14] is that the previous ones were constructed using decision algorithms of joinability and reachability, but the present approach uses only a decision algorithm of joinability for confluent semiconstructor TRSs [10], since the reachability problem is undecidable [11]. Besides, complex typed pairs of terms used in the previous ones are changed to simplified typed pairs which are used in the present one. Moreover, using this new result we give a sufficient condition for ensuring the decidability of the unification problem for a new subclass of nonlinear TRSs that are different from semi-constructor TRSs. As other known results for non-right-linear TRSs, the unification problem is decidable for shallow TRSs [2] and semi-linear TRSs [6].

\section{Preliminaries}

We assume that the reader is familiar with standard definitions of rewrite systems (see [1], [16]) and we just recall here main notations used in this paper.

We use $\varepsilon$ to denote the empty string and $\emptyset$ to denote the empty set. For a Set $A$, let $\mathcal{P}(A)$ be the set of all subsets of $A$, and let $|A|$ be the cardinality of $A$. Let $\mathbf{N}$ be the set of nonnegative integers. For any elements $a, b \in A$, mapping $\phi: A \rightarrow B$, and partial or proper order $>$ on $B$, we write $a>_{\phi} b$ if $\phi(a)>\phi(b)$ and $a={ }_{\phi} b$ if $\phi(a)=\phi(b)$.

Let $X$ be a set of variables, $F$ a finite set of function symbols graded by an arity function ar: $F \rightarrow \mathbf{N}, F_{n}=\{f \in$ $F \mid \operatorname{ar}(f)=n\}$ and $T$ the set of terms constructed from $X$ and $F$. We use $x, y, z$ as variables, $c, d$ as constant symbols, $f$, $g$ as function symbols of non-zero arity, and $r, s, t$ as terms. Let Leaf $=X \cup F_{0}$. Each element in Leaf is called a leaf 
symbol. A term is ground if it has no variable. Let $G$ be the set of ground terms, and let $S=T \backslash(G \cup X)$. Let $\mathrm{V}(s)$ be the set of variables occurring in $s$. The root symbol is defined as $\operatorname{root}(a)=a$ if $a$ is a leaf symbol and $\operatorname{root}\left(f\left(t_{1}, \ldots, t_{n}\right)\right)=f$.

A position in a term is expressed by a sequence of positive integers. Let $O(s)$ be the set of positions of $s$. We use $u, v$ as positions. Positions are partially ordered by the prefix ordering $\leq$. To denote that positions $u$ and $v$ are disjoint, we use $u \mid v$. For a set of positions $W$, the set of all minimal positions (w.r.t. $\leq$ ) of $W$ is denoted by $\operatorname{Min}(W)$.

Let $s_{\mid u}$ be the subterm of $s$ at position $u$. Let $\operatorname{Psub}(s)$ be the set of proper subterms of $s: \operatorname{Psub}(s)=\left\{s_{\mid u} \mid u \in\right.$ $\mathcal{O}(s) \backslash\{\varepsilon\}\}$. The domain $T$ of Psub is extended to $\mathcal{P}(T)$, i.e., $\operatorname{Psub}\left(T^{\prime}\right)=\bigcup_{s \in T^{\prime}} \operatorname{Psub}(s)$. We use $s[t]_{u}$ to denote the term obtained from $s$ by replacing the subterm $s_{\mid u}$ by $t$. For a sequence $\left(u_{1}, \cdots, u_{n}\right)$ of pairwise disjoint positions and terms $r_{1}, \cdots, r_{n}$, we use $s\left[r_{1}, \cdots, r_{n}\right]_{\left(u_{1}, \ldots, u_{n}\right)}$ to denote the term obtained from $s$ by replacing each subterm $s_{\mid u_{i}}$ by $r_{i}(1 \leq i \leq n)$.

A rewrite rule is defined as a directed equation $\alpha \rightarrow \beta$ such that $\alpha \notin X$ and $\mathrm{V}(\alpha) \supseteq \mathrm{V}(\beta)$. A TRS $R$ is a finite set of rewrite rules. We write $s \stackrel{u}{\rightarrow} R$ when there exist $r$, a substitution $\sigma$ and $\alpha \rightarrow \beta \in R$ that satisfy $s=r[\alpha \sigma]_{u}$ and $t=r[\beta \sigma]_{u}$. Position $u$ is called a redex position. If $u$ and $R$ are clear from the context, we can drop them. Let $\leftarrow$ be the inverse of $\rightarrow, \leftrightarrow=\rightarrow \cup \leftarrow$ and $\downarrow=\rightarrow^{*} \cdot \leftarrow^{*}$. Let $\gamma: s_{1} \stackrel{u_{1}}{\leftrightarrow} s_{2} \ldots \stackrel{u_{n-1}}{\leftrightarrow} s_{n}$ be a rewrite sequence. This sequence is abbreviated to $\gamma: s_{1} \leftrightarrow^{*} s_{n}$ and $\mathcal{R}(\gamma)=\left\{u_{1}, \cdots, u_{n-1}\right\}$ is the set of the redex positions of $\gamma$. For $v \in O\left(s_{1}\right)$, if $u>v$ or $u \mid v$ for all $u \in \mathcal{R}(\gamma)$, then $\gamma$ is called $v$-invariant. For a set of positions $W$, if $u \geq v$ or $u \mid v$ for all $u \in \mathcal{R}(\gamma)$ and $v \in W$ then $\gamma$ is called $W$-frontier. For any sequence $\gamma$ and position set $W, \mathcal{R}(\gamma) \geq W$ if for any $v \in \mathcal{R}(\gamma)$ there exists a $u \in W$ such that $v \geq u$. If $\mathcal{R}(\gamma) \geq W$, we write $\gamma: s_{1} \stackrel{\gtrless W}{\leftrightarrow} s_{n}$.

Let $\mathcal{O}_{G}(s)$ be the set of positions of $s$ at which the subterms are ground: $O_{G}(s)=\left\{u \in O(s) \mid s_{\mid u} \in G\right\}$. For any set $\Delta \subseteq X \cup F$, let $O_{\Delta}(s)=\left\{u \in O(s) \mid \operatorname{root}\left(s_{\mid u}\right) \in \Delta\right\}$. Let $O_{x}(s)=O_{\{x\}}(s)$. The set $D_{R}$ of defined symbols for a TRS $R$ is defined as $D_{R}=\{\operatorname{root}(\alpha) \mid \alpha \rightarrow \beta \in R\}$. If $R$ is clear from the context, we can drop $R$. A term $s$ is semi-constructor if for each defined symbol occurring in $s$ all the occurrences occur in ground subterms of $s$.

Definition 2.1: A rule $\alpha \rightarrow \beta$ is ground if $\alpha \in G$, right-ground if $\beta \in G$, and semi-constructor if $\beta$ is semiconstructor. A TRS $R$ is right-ground if every rule in $R$ is right-ground, and semi-constructor if every rule in $R$ is semi-constructor. A TRS $R$ is confluent if $\leftarrow_{R}^{*} \cdot \rightarrow_{R}^{*} \subseteq \downarrow_{R}$. A TRS $R$ is $C R$ if $\leftrightarrow_{R}^{*} \subseteq \downarrow_{R}$. It is known that confluence and CR are equivalent.

Example 2.2: $\quad$ Let $R_{\mathrm{e}}=\{\operatorname{nand}(x, x) \rightarrow \neg(\wedge(x, x))$, $\operatorname{nand}(\neg(\wedge(x, x)), x) \rightarrow \mathrm{t}, \mathrm{t} \rightarrow \operatorname{nand}(\mathrm{f}, \mathrm{f}), \mathrm{f} \rightarrow \operatorname{nand}(\mathrm{t}, \mathrm{t})\}$. Note that the set of defined symbols $D_{R_{\mathrm{e}}}$ is $\{$ nand, $\mathrm{t}, \mathrm{f}\} . R_{\mathrm{e}}$ is semi-constructor, non-terminating and confluent [4].

Definition 2.3: We use $s \approx t$ to denote the pair of terms $s$ and $t . s \approx t$ is joinable for a TRS $R$ if $s \downarrow_{R} t . s \approx t$ is unifiable modulo a TRS $R$ (or simply $R$-unifiable) if there exist a substitution $\theta$ and a rewrite sequence $\gamma$ such that $\gamma$ : $s \theta \leftrightarrow^{*} t \theta$. Such $\theta$ and $\gamma$ are called an $R$-unifier and a proof of $s \approx t$, respectively. This notion is extended to sets of term pairs: for $\Gamma \subseteq T \times T, \theta$ is an $R$-unifier of $\Gamma$ if $\theta$ is an $R$-unifier of every pair $s \approx t$ of $\Gamma$. In this case, $\Gamma$ is $R$-unifiable. As a special case of $R$-unifiability, $s \approx t$ is $\emptyset$-unifiable if there exists a substitution $\theta$ such that $s \theta=t \theta$, i.e., $\emptyset$-unifiability coincides with usual unifiability.

We use $\{\cdots\}_{\mathrm{m}}$ to denote a multiset. Let $\ll$ be the multiset extension of usual relation $<$ on $N$, and $\ll$ be $\ll U=$. We use $\sqcup$ to denote multiset union.

Definition 2.4: For a term $t$, we define the height of $t$ as follows.

(1)

$$
\operatorname{height}(t)=\left\{\begin{array}{l}
1+\max \left\{\text { height }\left(t_{i}\right) \mid 1 \leq i \leq n\right\} \\
\left(\text { if } t=f\left(t_{1}, \cdots, t_{n}\right), n>0\right) \\
0 \text { (if } t \in \text { Leaf })
\end{array}\right.
$$

(2) For $B \in\left\{F_{0}, G\right\}$, we define $\mathrm{hD}_{B}(t)$ as follows.

$$
\mathrm{hD}_{B}(t)=\left\{\begin{array}{l}
w_{f}+\max \left\{\mathrm{hD}_{B}\left(t_{i}\right) \mid 1 \leq i \leq n\right\} \\
\left(\text { if } t=f\left(t_{1}, \cdots, t_{n}\right), n>0\right) \\
0(\text { if } t \in X \cup B)
\end{array}\right.
$$

Here, $w_{f}=1+2 \max \{$ height $(\beta) \mid \alpha \rightarrow \beta \in R\}$ if $f$ is a defined symbol for TRS $R$, otherwise $w_{f}=1$. In this function, every subterm belonging to $X \cup B$ is not counted.

(3) For $B \in\left\{F_{0}, G\right\}$, we define $\mathrm{HD}_{B}(t)=\left\{\mathrm{hD}_{B}\left(t_{\mid u}\right) \mid u \in\right.$ $\left.O(t) \backslash O_{B}(t)\right\}_{\mathrm{m}}$, which is the multiset of the $\mathrm{hD}_{B}$-values of all subterms of $t$ except elements of $B$.

Example 2.5: For TRS $\mathrm{M}\left(R_{\mathrm{e}}\right)$ of Example Appendix B.3 in Appendix $\mathrm{B}, \mathrm{hD}_{F_{0}}(x)=\mathrm{hD}_{G}(x)=0$, $\mathrm{hD}_{F_{0}}(\operatorname{nand}(x, \neg(\mathrm{t})))=6, \mathrm{hD}_{G}(\operatorname{nand}(x, \neg(\mathrm{t})))=5$, $\mathrm{HD}_{F_{0}}(x)=\operatorname{HD}_{G}(x)=\{0\}_{\mathrm{m}}, \operatorname{HD}_{F_{0}}(\operatorname{nand}(x, \neg(\mathrm{t})))=$ $\{0,1,6\}_{\mathrm{m}}$, and $\mathrm{HD}_{G}(\operatorname{nand}(x, \neg(\mathrm{t})))=\{0,5\}_{\mathrm{m}}$.

For the measure $\mathrm{HD}_{B}$, the following lemma holds.

Lemma 2.6: For any $s, t$, the following conditions hold.

(1) If $s<\mathrm{hD}_{B} t$ then $s \ll \mathrm{HD}_{B} t$.

(2) If $s \ll_{\mathrm{HD}_{B}} t$ then $s \leq_{\mathrm{hD}_{B}} t$

(3) For any $r$ and $u \in O(r)$, if $s \ll \mathrm{HD}_{B} t$ then $r[s]_{u} \ll \mathrm{HD}_{B}$ $r[t]_{u}$.

\section{Proof}

(1) For any subterm $s^{\prime}$ of $s, s^{\prime} \leq_{\mathrm{hD}_{B}} s$. By $s<{ }_{\mathrm{hD}_{B}} t, s \ll_{\mathrm{HD}_{B}}$ $t$ holds.

(2) To the contrary, we assume that $s>_{\mathrm{hD}_{B}}$ t. By (1), $s \gg_{\mathrm{HD}_{B}} t$, a contradiction.

(3) Let $\hat{s}=f\left(r_{1}, \cdots, r_{i-1}, s, r_{i+1}, \cdots, r_{n}\right)$ and $\hat{t}=$ $f\left(r_{1}, \cdots, r_{i-1}, t, r_{i+1}, \cdots, r_{n}\right)$ where $f \in F_{n}$ and $i \in$ $\{1, \cdots, n\}$. It suffices to show that $\left\{\operatorname{hD}_{B}(\hat{s})\right\}_{\mathrm{m}} \sqcup$ $\mathrm{HD}_{B}(s) \ll\left\{\mathrm{hD}_{B}(\hat{t})\right\}_{\mathrm{m}} \sqcup \mathrm{HD}_{B}(t)$. By (2), $s \leq_{\mathrm{hD}_{B}} t$, so $\hat{s} \leq \mathrm{hD}_{B} \hat{t}$ holds. If $\hat{s}<\mathrm{hD}_{B} \hat{t}$ then $\hat{s} \ll \mathrm{HD}_{B} \hat{t}$ holds by (1). If $\hat{s}=\mathrm{hD}_{B} \hat{t}$ then $\hat{s} \ll \mathrm{HD}_{B} \hat{t}$ holds by $s \ll \mathrm{HD}_{B} t$. 


\section{Basic Results}

In order to show the decidability of unification for confluent semi-constructor TRSs, we need the algorithm deciding the joinability problem in [10] and some definitions and lemmata in [10]. We describe these definitions and results (without the proofs) in this section.

\subsection{Standard Semi-Constructor TRSs}

We use $R_{\mathrm{rg}}$ and $R_{\mathrm{nrg}}$ to denote the sets of right-ground and non-right-ground rewrite rules in TRS $R$, respectively. That is, $R=R_{\mathrm{rg}} \cup R_{\mathrm{nrg}}$.

Definition 3.1: [10] A TRS $R$ is standard if for every $\alpha \rightarrow$ $\beta \in R$, either $\alpha \in F_{0}$ and height $(\beta) \leq 1$ or $\alpha \notin F_{0}$ and $O_{G}(\beta) \subseteq O_{F_{0}}(\beta)$ holds. Note that for any right ground rule $\alpha \rightarrow \beta$ in a standard TRS, $\alpha \in F_{0}$ and height $(\beta) \leq 1$ or $\alpha \notin F_{0}$ and $\beta \in F_{0}$ hold.

Let $R$ be a confluent semi-constructor TRS. We have introduced an effectively computable function $S$ which takes TRS $R$ and produces standard TRS S $(R)$ in [10]..$^{\dagger}$ We have shown that $\mathrm{S}(R)$ is standard, confluent and semi-constructor. The following lemma also holds.

Lemma 3.2: For any confluent semi-constructor TRS $R$ and terms $s, t$ which do not contain any new constant generated by $\mathrm{S}, s \approx t$ is $R$-unifiable iff $s \approx t$ is $\mathrm{S}(R)$-unifiable.

We can assume that a given confluent semi-constructor TRS is standardized, hereafter.

\subsection{Shortcut Rules and Quasi-Standard Semi-Constructor TRSs}

We add new ground rules called shortcut rules to standard TRS $R$, and obtain TRS $R^{\prime}$ satisfying that two constants are joinable in $R$ iff they are joinable by only right-ground rules of $R^{\prime}$. Right-hand sides of added shortcut rules may have height greater than 1 . These rules are called type $\mathrm{C}$ rules and defined as follows.

Definition 3.3: [10]

(1) For TRS $R$, a rule $\alpha \rightarrow \beta \in R$ has type $\mathrm{C}$ if $\alpha \in F_{0}$, $\beta \notin F_{0}$, and $O_{D_{R}}(\beta) \subseteq O_{F_{0}}(\beta)$. Let $R_{\mathrm{C}}$ be the set of type $\mathrm{C}$ rules in $R$.

(2) A TRS $R$ is quasi-standard if $R \backslash R_{\mathrm{C}}$ is standard.

Henceforth, we assume that $R$ is confluent, quasi-standard, and semi-constructor. To describe how to produce shortcut rules, we need some preliminaries.

Definition 3.4: [10] Let $\operatorname{Bud}\left(R_{\mathrm{C}}\right)=F_{0} \cup \operatorname{Psub}(\{\beta \mid \alpha \rightarrow$ $\left.\beta \in R_{\mathrm{C}}\right\}$ ).

Lemma 3.5: [10] For any rewrite sequence $\gamma: s \rightarrow_{R_{\mathrm{rg}}}^{*} t$ and $u \in O(t)$, if there exists $v \in \mathcal{R}(\gamma)$ such that $v<u$, then there exists $s^{\prime} \in \operatorname{Bud}\left(R_{\mathrm{C}}\right)$ such that $s \rightarrow_{R_{\mathrm{rg}}}^{*} t\left[s^{\prime}\right]_{u}$ and $s^{\prime} \rightarrow{ }_{R_{\mathrm{rg}}}^{*} t_{\mid u}$.
Definition 3.6: [10]

(1) The function linearize $(s)$ linearizes non-linear term $s$ as follows. For each variable occurring more than once in $s$, the first occurrence is not renamed, and the other ones are replaced by new pairwise distinct variables. For example, linearize $(\operatorname{nand}(x, x))=\operatorname{nand}\left(x, x_{1}\right)$. If function linearize replaces $x$ by $x_{1}$ then we use $x \equiv x_{1}$ to denote the replacement relation.

(2) A substitution $\sigma$ is joinability preserving under relation $\equiv$ for TRS $R_{\mathrm{rg}}$ if $x \sigma \downarrow_{R_{\mathrm{rg}}} x^{\prime} \sigma$ whenever $x \equiv x^{\prime}$.

(3) A substitution $\sigma: \mathrm{V}\left(t^{\prime}\right) \rightarrow \operatorname{Psub}(s) \cup \operatorname{Bud}\left(R_{\mathrm{C}}\right)$ is a bud substitution for $s$ and $t$, where $t^{\prime}=\operatorname{linearize}(t)$, if $s \rightarrow{ }_{R_{\mathrm{rg}}}^{*} t^{\prime} \sigma$ and $\sigma$ is joinability preserving under relation $\equiv$ for $R_{\mathrm{rg}}$. Note that if $s$ is ground then $t^{\prime} \sigma$ is ground. Let $\operatorname{BudMap}_{R}(s, t)$ be the set of such bud substitutions.

Lemma 3.7: [10] Let $\alpha \rightarrow \beta \in R_{\mathrm{nrg}}$ and $\gamma: s \rightarrow_{R_{\mathrm{rg}}}^{*} \alpha \theta$ for some $\theta$. Then, there exists $\sigma \in \operatorname{BudMap}_{R}(s, \alpha)$ such that $s \rightarrow{ }_{R_{\mathrm{rg}}}^{*} \alpha^{\prime} \sigma \rightarrow{ }_{R_{\mathrm{rg}}}^{*} \alpha \theta$ and $\beta \sigma \rightarrow{ }_{R_{\mathrm{rg}}}^{*} \beta \theta$ where $\alpha^{\prime}=$ linearize $(\alpha)$.

By Lemma 3.7, for any constant $d$ and rewrite sequence $d \rightarrow_{R_{\mathrm{rg}}}^{*} \alpha \theta \rightarrow_{R_{\mathrm{nrg}}} \beta \theta$, there exists $\alpha^{\prime} \sigma$ such that $d \rightarrow_{R_{\mathrm{rg}}}^{*}$ $\alpha^{\prime} \sigma \rightarrow_{R_{\mathrm{rg}}}^{*} \alpha \theta$ and $\beta \sigma \rightarrow_{R_{\mathrm{rg}}}^{*} \beta \theta$ where $\alpha^{\prime}=$ linearize $(\alpha)$. So, we have $d \rightarrow_{R^{\prime}}^{*} \beta \theta$ for $R^{\prime}=R_{\mathrm{rg}} \cup\{d \rightarrow \beta \sigma\}$. Thus, by adding shortcut rules such as $d \rightarrow \beta \sigma$, we can remove applications of the non-right-ground rule $\alpha \rightarrow \beta$. Note that confluence and joinability properties are preserved even if we add $d \rightarrow \beta \sigma$ since $d \downarrow_{R} \beta \sigma$. However, shortcut rules may be added infinitely in this procedure. To avoid this, we apply a procedure which bounds the number of shortcut rules. We have introduced an effectively computable function $\mathrm{M}$ to implement this procedure in $[10]^{\dagger \dagger}$ and shown that $\mathrm{M}(R)$ is confluent, quasi-standard and semi-constructor. Moreover, we have shown that the following lemma holds for $\mathrm{M}(R)$.

Lemma 3.8: [10]

(1) For any $d$ and $s$, if $d \rightarrow_{R}^{*} s$ then $d \rightarrow_{\mathrm{M}(R)_{\mathrm{rg}}}^{*} s$.

(2) $\rightarrow{ }_{\mathrm{M}(R)} \subseteq \downarrow_{R}$.

\subsection{Auxiliary Terms}

Let $s$ be a ground term.

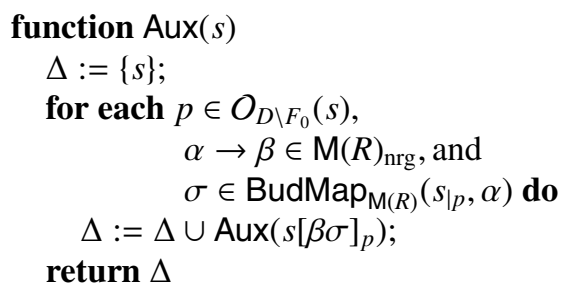

Example 3.9: For TRS $\mathrm{M}\left(R_{\mathrm{e}}\right)$ of Example Appendix B.3, $\operatorname{Aux}(\neg(\operatorname{nand}(\mathrm{t}, \mathrm{t})))=\{\neg(\operatorname{nand}(\mathrm{t}, \mathrm{t})), \neg(\neg(\wedge(\mathrm{t}, \mathrm{t})))\}$.

\footnotetext{
${ }^{\dagger}$ This function $\mathrm{S}$ is given in Appendix $\mathrm{A}$ of this paper.

${ }^{\dagger \dagger}$ This function $\mathrm{M}$ is given in Appendix $\mathrm{B}$ of this paper.
} 
We have shown that $\operatorname{Aux}(s)$ is finite and computable.

Lemma 3.10: [10] For any ground term $s$,

(1) For any $s^{\prime} \in \operatorname{Aux}(s), s^{\prime}$ is a ground term and $s^{\prime} \downarrow_{\mathrm{M}(R)} s$.

(2) If $s \rightarrow_{R}^{*} t$ then there exists $s^{\prime} \in \operatorname{Aux}(s)$ such that $s^{\prime} \rightarrow_{\mathrm{M}(R)_{\mathrm{rg}}}^{*} t$.

We call $s^{\prime}$ in Lemma 3.10 (2) an auxiliary term of $(s, t)$. Using this term, we can transform non-right-ground rewrite sequences to right-ground rewrite sequences.

Example 3.11: For the rewrite sequence $\neg($ nand $(\mathrm{t}, \mathrm{t})) \rightarrow{ }_{R_{\text {erg }}}^{*} \neg($ nand $($ nand $(\mathrm{f}, \mathrm{f})$, nand $(\mathrm{f}, \mathrm{f})))$ $\rightarrow R_{\mathrm{e}} \neg(\neg(\wedge($ nand $(\mathrm{f}, \mathrm{f})$, nand $(\mathrm{f}, \mathrm{f}))))$, we can choose $\neg(\neg(\wedge(\mathrm{t}, \mathrm{t}))) \in \operatorname{Aux}(\neg($ nand $(\mathrm{t}, \mathrm{t})))$ and $\neg(\neg(\wedge(\mathrm{t}, \mathrm{t}))) \rightarrow_{\mathrm{M}\left(R_{\mathrm{e}}\right)_{\mathrm{rg}}} \neg(\neg(\wedge($ nand $(\mathrm{f}, \mathrm{f})$, nand $(\mathrm{f}, \mathrm{f}))))$.

Lemma 3.12: For any confluent standard semi-constructor TRS $R, s \approx t$ is $R$-unifiable iff $s \approx t$ is $\mathrm{M}(R)$-unifiable.

Proof Only if part: Since $R$ is confluent, there exists $\theta$ such that $s \theta \downarrow_{R} t \theta$. W.l.o.g., we can assume that $s \theta$ and $t \theta$ are ground terms. By Lemma 3.10(2), there exist $s^{\prime} \in \operatorname{Aux}(s \theta), t^{\prime} \in \operatorname{Aux}(t \theta)$ such that $s^{\prime} \downarrow_{\mathrm{M}(R)_{\mathrm{rg}}} t^{\prime}$. By Lemma $3.10(1), s \theta \leftrightarrow_{\mathrm{M}(R)}^{*} t \theta$.

If part: There exists $\theta$ such that $s \theta \leftrightarrow_{\mathrm{M}(R)}^{*} t \theta$. By Lemma $3.8(2), s \theta \leftrightarrow_{R}^{*} t \theta$.

In this paper, we give an $R$-unification algorithm for confluent semi-constructor TRSs. By Lemma 3.12, we assume that confluent semi-constructor TRS $R$ is quasistandard and an output of Algorithm $\mathrm{M}$, that is, $\mathrm{M}(R)=R$ holds.

\section{Locally Minimum Unifiers and Typed Pairs of Terms}

In this section, we introduce the notions of locally minimum unifiers and typed pairs of terms for our unification algorithm.

\section{Definition 4.1:}

(1) Let \# $(t)=\left(\mathrm{HD}_{F_{0}}(t), \operatorname{ord}(t)\right)$, where ord : $T \rightarrow \mathbf{N}$ is an injective mapping. We use lexicographic ordering $>_{\#}$ to compare any pair of terms. We assume that if $s>_{\#} t$ then $r[s]_{u}>_{\#} r[t]_{u}$ for any $r, s, t$. The existence of such an effectively computable function ord is shown in Appendix A.2 of [10] for ground terms. We can easily extend this function to one for non-ground terms [8].

(2) Let $\mathcal{L}(t)=\left\{s \mid s \leftrightarrow^{*} t\right\}$. Note that it is decidable for any terms $s$ and $s^{\prime}$, whether $s^{\prime} \in \mathcal{L}(s)$ holds or not [10].

(3) $s_{0}$ is minimum if $s_{0}$ is minimum in $\mathcal{L}\left(s_{0}\right)$ on $>_{\#}$.

Lemma 4.2: For any minimum term, its subterm is minimum.

This proof is obvious, since if $s>_{\#} t$ then $r[s]_{u}>_{\#} r[t]_{u}$ for any $r, s, t$, so that if $r$ is minimum then $r_{\mid u}$ must be minimum.

Lemma 4.3: Let $s_{0}$ be minimum and $\gamma: s_{0} \rightarrow^{*} t$. Then, $\mathcal{R}(\gamma) \geq O_{\text {Leaf }}\left(s_{0}\right)$. (That is, only leaf symbols of $s_{0}$ are rewritten in $\gamma$.)

Proof We show by induction on $\mathrm{HD}_{F_{0}}\left(s_{0}\right)$. It is trivial in case of $\mathrm{HD}_{F_{0}}\left(s_{0}\right)=\emptyset$ or $\{0\}_{\mathrm{m}}$. So, we consider the case $\mathrm{HD}_{F_{0}}\left(s_{0}\right) \gg\{0\}_{\mathrm{m}}$. If $\gamma$ is $\varepsilon$-invariant then this lemma holds by the induction hypothesis. Thus, it is sufficient to show that $\gamma$ is $\varepsilon$-invariant. We assume to the contrary that $s_{0} \stackrel{>\varepsilon}{\rightarrow}{ }^{*}$ $\alpha \theta \stackrel{\mathcal{\varepsilon}}{\rightarrow} \beta \theta$ for some rule $\alpha \rightarrow \beta$ and substitution $\theta$. Then, the following conditions (a)-(c) hold: (a) $\operatorname{root}\left(s_{0}\right) \in D$ and $\alpha \notin F_{0}$, (b) $\beta \notin G$, i.e., $\alpha \rightarrow \beta \in R_{\text {nrg }}$, (c) $s_{0} \rightarrow_{R_{\text {rg }}}^{*} \alpha \theta$. The proof of (a) is obvious by $\mathrm{HD}_{F_{0}}\left(s_{0}\right) \gg\{0\}_{\mathrm{m}}$. (b) holds, since if $\beta \in G$ then we have $\beta \in F_{0}$ by quasi-standardness, which contradicts that $s_{0}$ is minimum. To show (c), let $\delta: s_{0} \stackrel{>\varepsilon}{\rightarrow}^{*}$ $\alpha \theta$. By the induction hypothesis, $\mathcal{R}(\delta) \geq O_{\text {Leaf }}\left(s_{0}\right)$, so that $s_{0} \rightarrow{ }_{R_{\mathrm{rg}}}^{*} \alpha \theta$ by Lemma 3.8 (1) and $\mathrm{M}(R)=R$. Thus, (c) holds. By (b), (c) and Lemma 3.7, there exists a substitution $\sigma \in \operatorname{BudMap}_{R}\left(s_{0}, \alpha\right)$ such that $s_{0} \rightarrow{ }_{R_{\mathrm{rg}}}^{*} \alpha^{\prime} \sigma \rightarrow_{R_{\mathrm{rg}}}^{*} \alpha \theta$ and $\beta \sigma \rightarrow_{R_{\mathrm{rg}}}^{*} \beta \theta$ where $\alpha^{\prime}=$ linearize $(\alpha)$. Hence, $s_{0} \downarrow \beta \sigma$ holds. Since $\operatorname{root}\left(s_{0}\right) \in D$ and $\beta$ is semi-constructor, $\mathrm{hD}_{F_{0}}\left(s_{0}\right)=$ $1+2 \max \{\operatorname{height}(\beta) \mid \alpha \rightarrow \beta \in R\}+\max \left\{\mathrm{hD}_{F_{0}}\left(s_{\mid i}\right) \mid 1 \leq i \leq\right.$ $\left.\operatorname{ar}\left(\operatorname{root}\left(s_{0}\right)\right)\right\}>\mathrm{hD}_{F_{0}}(\beta \sigma)$. By Lemma 2.6 (1) $s_{0} \gg_{\mathrm{HD}_{F_{0}}} \beta \sigma$ holds. This contradicts that $s_{0}$ is minimum.

Example 4.4: Terms $\mathrm{t}$ and $\operatorname{nand}(\mathrm{t}, x)$ are minimum. $O_{\text {Leaf }}(\operatorname{nand}(\mathrm{t}, x))=\{1,2\}$. Only leaf symbols of nand $(\mathrm{t}, x)$ are rewritten in a rewrite sequence such as nand(t, $x) \stackrel{1}{\rightarrow}$ $\operatorname{nand}(\operatorname{nand}(\mathrm{f}, \mathrm{f}), x) \stackrel{11}{\rightarrow} \operatorname{nand}(\operatorname{nand}(\operatorname{nand}(\mathrm{t}, \mathrm{t}), \mathrm{f}), x) \stackrel{111}{\rightarrow} \cdots$.

Lemma 4.5: The minimum term in $\mathcal{L}(s)$ is computable. Proof Let $s_{0}$ be the minimum term in $\mathcal{L}(s)$. First we show that $\mathrm{V}\left(s_{0}\right) \subseteq \mathrm{V}(s)$. By confluence of $R$, there exists some term $r$ such that $s_{0} \rightarrow^{*} r$ and $s \rightarrow^{*} r$. By Lemma 4.3, only leaf symbols of $s_{0}$ are rewritten, so that $\mathrm{V}\left(s_{0}\right)=\mathrm{V}(r)$. Thus, $\mathrm{V}\left(s_{0}\right) \subseteq \mathrm{V}(s)$ as claimed. The set $\left\{s^{\prime} \mid s^{\prime} \leq_{\#} s, \mathrm{~V}\left(s^{\prime}\right) \subseteq \mathrm{V}(s)\right\}$ is finite. Since joinability is decidable, $s_{0}$ is computable.

\section{Definition 4.6:}

(1) A substitution $\theta$ is a locally minimum substitution if $x \theta$ is minimum for every $x \in \operatorname{Dom}(\theta)$.

(2) Let $\Gamma \subseteq T \times T$. A locally minimum substitution $\theta$ is a locally minimum $R$-unifier of $\Gamma$ if $\theta$ is an $R$-unifier of $\Gamma$.

Our unification algorithm takes a pair $s \approx t$ as input and produces a locally minimum unifier $\theta$ of $s \approx t$ iff $s \approx t$ is $R$-unifiable. Different types of pairs are distinguished by using the notation $s \triangleright t$ and $s \approx_{\mathrm{vf}} t$, which are said to be of type $\triangleright$ and of type vf, respectively. These definitions are similar to those of [14]. Type $\triangleright_{U}$ was used in [14], but the parameter $U$ is not essential, so omitted.

Definition 4.7: Let $E_{0}=\left\{s \approx t, s \approx_{\mathrm{vf}} t\right.$, fail $\mid s, t \in$ $T\} \cup\{s \triangleright t \mid s \in T, t \in S\}$. Here, fail is introduced as a special symbol and we assume that there exists no $R$-unifier of fail [14]. For $\Gamma \subseteq E_{0}$ and substitution $\theta$, let $\Gamma \theta=\{s \theta \approx$ $t \theta \mid s \approx t \in \Gamma$ or $s \triangleright t \in \Gamma$ and $t \theta \notin S\} \cup\{s \theta \triangleright t \theta \mid s \triangleright t \in$ $\Gamma$ and $t \theta \in S\} \cup\left\{s \theta \approx_{\mathrm{vf}} t \theta \mid s \approx_{\mathrm{vf}} t \in \Gamma\right\} \cup\{$ fail $\mid$ fail $\in \Gamma\}$.

$R$-unifiers of these new pairs are required to satisfy additional conditions derived from these types. 
Definition 4.8: A substitution $\theta$ is a (locally minimum) $R$ unifier of $s \triangleright t$ if $\theta$ is a (locally minimum) $R$-unifier of $s \approx t$ and there exists a rewrite sequence $\gamma: s \theta \rightarrow^{*} r \stackrel{\geq O_{X}(t)}{\leftrightarrow} t \theta$ for some term $r$. A substitution $\theta$ is a (locally minimum) $R$-unifier of $s \approx_{\mathrm{vf}} t$ if $\theta$ is a (locally minimum) $R$-unifier of $s \approx t$ and there exists $\gamma: s \theta \leftrightarrow^{*} t \theta$, where $\gamma$ is $O_{X}(t)$-frontier.

Note that if $t \in G$ then $\theta$ is an $R$-unifier of $s \approx_{\mathrm{vf}} t$ iff $\theta$ is that of $s \approx t$.

Example 4.9: Let $\mathrm{M}\left(R_{\mathrm{e}}\right)$ be the TRS of Example Appendix B.3.

1. nand $(\mathrm{f}, \neg(\operatorname{nand}(\mathrm{t}, \mathrm{t}))) \triangleright \operatorname{nand}(y, \neg(y))$ is $\mathrm{M}\left(R_{\mathrm{e}}\right)$-unifiable, since any substitution $\theta$ satisfying $y \theta=f$ is an $\mathrm{M}\left(R_{\mathrm{e}}\right)$-unifier: nand $(\mathrm{f}, \neg($ nand $(\mathrm{t}, \mathrm{t}))) \stackrel{21}{\leftarrow} \operatorname{nand}(\mathrm{f}, \neg(\mathrm{f}))$.

2. $\operatorname{nand}(\mathrm{t}, \operatorname{nand}(\mathrm{t}, \mathrm{t})) \approx_{\mathrm{vf}} \operatorname{nand}(\operatorname{nand}(\mathrm{f}, \mathrm{f}), y)$ is $\mathrm{M}\left(R_{\mathrm{e}}\right)$-unifiable, since any substitution $\theta$ satisfying $y \theta=\mathrm{f}$ is an $\mathrm{M}\left(R_{\mathrm{e}}\right)$-unifier: nand $(\mathrm{t}, \operatorname{nand}(\mathrm{t}, \mathrm{t})) \stackrel{1}{\rightarrow} \operatorname{nand}(\operatorname{nand}(\mathrm{f}, \mathrm{f})$, $\operatorname{nand}(\mathrm{t}, \mathrm{t}) \stackrel{2}{\leftarrow} \operatorname{nand}($ nand $(\mathrm{f}, \mathrm{f}), \mathrm{f})$.

To convert typed pairs into the untyped ones, we define the following function Core.

Definition 4.10: [14] For $\Gamma \subseteq E_{0}$, let Core $(\Gamma)=\{s \approx t \mid s \approx$ $t \in \Gamma$ or $s \triangleright t \in \Gamma$ or $\left.s \approx_{\mathrm{vf}} t \in \Gamma\right\} \cup\{$ fail $\mid$ fail $\in \Gamma\}$.

The following definition and technical lemma is needed to show the validity of TT transformation of Stage I of our unification algorithm described in Sect. 5 .

Definition 4.11: A substitution $\sigma: \mathrm{V}\left(t^{\prime}\right) \rightarrow \operatorname{Psub}(s) \cup$ $\operatorname{Bud}\left(R_{\mathrm{C}}\right) \cup \mathrm{V}\left(t^{\prime}\right)$ is an abstract one of $\sigma^{\prime} \in \operatorname{BudMap}_{R}(s \theta, t)$ if the following condition holds: $x \sigma=x$ if $x \sigma^{\prime} \in \operatorname{Psub}(y \theta) \cup$ $\{y \theta\}, x \sigma=s_{\mid v}$ if $x \sigma^{\prime}=s_{\mid v}$ for some $v \in O(s), x \sigma=x \sigma^{\prime}$ if $x \sigma^{\prime} \in \operatorname{Psub}(s) \cup \operatorname{Bud}\left(R_{C}\right)$. Here, $t^{\prime}=$ linearize $(t)$. Let $\overline{\operatorname{BudMap}}_{R}(s, t)$ be the set of such substitutions.

Lemma 4.12: $\quad$ Let $s \in S$ and $U=O_{X}(s) \cup \operatorname{Min}\left(O_{G}(s)\right)$.

(1) Let $\gamma: s \theta \stackrel{\geq U}{\rightarrow} t, \theta$ is a locally minimum substitution, and $s_{\mid w} \rightarrow{ }_{R_{\mathrm{rg}}}^{*} t_{\mid w}$ holds for every $w \in \operatorname{Min}\left(O_{G}(s)\right)$. Then, for any $u \in O(t)$, there exists $s^{\prime} \in\left\{s_{\mid u} \theta\right\} \cup \operatorname{Bud}\left(R_{\mathrm{C}}\right) \cup$ $\left\{x \theta_{\mid v} \mid x \in \mathrm{V}(s), v \in O(x \theta)\right\}$ such that $s \theta \stackrel{\geq U}{\rightarrow} t\left[s^{\prime}\right]_{u}$ and $s^{\prime} \rightarrow^{*} t_{\mid u}$.

(2) Let $\alpha \rightarrow \beta \in R$ and $\gamma: s \theta \stackrel{\geq U}{\rightarrow} \alpha \sigma$ for some $\sigma$ $\theta$ is a locally minimum substitution and $s_{\mid v} \rightarrow_{R_{\mathrm{rg}}}^{*} t_{\mid v}$ holds for every $v \in \operatorname{Min}\left(O_{G}(s)\right)$. Then, there exist $\rho \in \overline{\operatorname{BudMap}}_{R}(s, \alpha)$ and a locally minimum substitution $\theta^{\prime}: \mathrm{V}(s) \cup \mathrm{V}\left(\alpha^{\prime}\right) \rightarrow \operatorname{Psub}(s \theta)$ such that $s \theta \stackrel{\geq U}{\rightarrow} \alpha^{\prime} \rho \theta^{\prime} \stackrel{\geq O_{X}\left(\alpha^{\prime}\right)}{\rightarrow} \alpha \sigma$ and $\beta \rho \theta^{\prime} \stackrel{\geq O_{X}(\beta)}{\rightarrow} \beta \sigma$ where $\alpha^{\prime}=$ linearize $(\alpha)$.

\section{Proof}

(1) Since $x \theta$ is minimum for every $x \in \mathrm{V}(s)$ and Lemma 4.3, there exists a sequence $\gamma: s \theta \stackrel{\geq U^{\prime}}{\rightarrow} t$, where $U^{\prime}=\left(O_{\text {Leaf }}(s \theta) \backslash O_{F_{0}}(s)\right) \cup \operatorname{Min}\left(O_{G}(s)\right)$. Thus, $u \geq v$ or $u<v$ for some $v \in U^{\prime}$ holds.

(a) Case of $u \geq v$ for some $v \in O_{\text {Leaf }}(s \theta) \backslash O_{F_{0}}(s)$ : $s \theta_{\mid v} \rightarrow{ }^{*} t_{\mid v}$ holds. If $u=v$ then we can choose $s \theta_{\mid v}$ as $s^{\prime}$. Otherwise, by $s \theta_{\mid v} \in F_{0}$ and Lemma 3.8(1), $s \theta_{\mid v} \rightarrow_{R_{\mathrm{rg}}}^{*} t_{\mid v}$ holds. By Lemma 3.5, there exists $s^{\prime \prime} \in$ $\operatorname{Bud}\left(R_{\mathrm{C}}\right)$ such that $s \theta_{\mid v} \rightarrow_{R_{\mathrm{rg}}}^{*}\left(t_{\mid v}\right)\left[s^{\prime \prime}\right]_{u^{\prime}}$ where $u=v u^{\prime}$ and $s^{\prime \prime} \rightarrow_{R_{\mathrm{rg}}}^{*} t_{u}$. Thus, we can choose $s^{\prime \prime}$ as $s^{\prime}$.

(b) Case of $u \geq v$ for some $v \in \operatorname{Min}\left(O_{G}(s)\right): s_{\mid v} \rightarrow_{R_{\mathrm{rg}}}^{*} t_{\mid v}$ holds. If $u=v$ or $s_{\mid v} \stackrel{\geq u^{\prime}}{\rightarrow} t_{\mid v}$, where $u=v u^{\prime}$ then we can choose $s \theta_{\mid u}$ as $s^{\prime}$. Otherwise, by Lemma 3.5, there exists $s^{\prime \prime} \in \operatorname{Bud}\left(R_{\mathrm{C}}\right)$ such that $s_{\mid v} \rightarrow_{R_{\mathrm{rg}}}^{*}\left(t_{\mid v}\right)\left[s^{\prime \prime}\right]_{u^{\prime}}$ where $u=v u^{\prime}$ and $s^{\prime \prime} \rightarrow_{R_{\mathrm{rg}}}^{*} t_{\mid u}$. Thus, we can choose $s^{\prime \prime}$ as $s^{\prime}$.

(c) Case of $u<v$ for some $v \in U^{\prime}: s \theta_{\mid u} \rightarrow^{*} t_{\mid u}$ holds. If $u \in O(s)$ then $s \theta_{\mid u}=s_{\mid u} \theta$ holds, so we can choose $s_{\mid u} \theta$ as $s^{\prime}$. Otherwise, there exists $x \in \mathrm{V}(s)$ such that $s \theta_{\mid u}=x \theta_{\mid u^{\prime}}$ for some $u^{\prime}$, so we can choose $x \theta_{\mid u^{\prime}}$ as $s^{\prime}$.

(2) Let $\left\{u_{1}, \cdots, u_{n}\right\}$ be $O_{X}(\alpha)$. For $u_{1}$, there exists $s_{1}^{\prime} \in$ $\left\{s_{\mid u_{1}} \theta\right\} \cup \operatorname{Bud}\left(R_{\mathrm{C}}\right) \cup\left\{x \theta_{\mid v} \quad \mid x \in \mathrm{V}(s), v \in O(x \theta)\right\}$ such that $s \theta \stackrel{\geq U}{\rightarrow} \alpha \sigma\left[s_{1}^{\prime}\right]_{u_{1}} \stackrel{\geq\left\{u_{1}\right\}}{\rightarrow} \alpha \sigma$ by (1). Let $\gamma_{1}: s \theta \stackrel{\geq U}{\rightarrow} \alpha \sigma\left[s_{1}^{\prime}\right]_{u_{1}}$. By similar arguments, there exists $s_{2}^{\prime} \in\left\{s_{\mid u_{2}} \theta\right\} \cup \operatorname{Bud}\left(R_{\mathrm{C}}\right) \cup\left\{x \theta_{\mid v} \mid x \in \mathrm{V}(s), v \in\right.$ $O(x \theta)\}$ such that $s \theta \stackrel{\geq U}{\rightarrow} \alpha \sigma\left[s_{1}^{\prime}\right]_{u_{1}}\left[s_{2}^{\prime}\right]_{u_{2}}$ and $s_{2}^{\prime} \rightarrow^{*}$ $\left(\alpha \sigma\left[s_{1}^{\prime}\right]_{u_{1}}\right)_{\mid u_{2}}$ for $\gamma_{1}$ by (1). By $u_{1} \mid u_{2}, \alpha \sigma\left[s_{1}^{\prime}\right]_{u_{1}}\left[s_{2}^{\prime}\right]_{u_{2}}=$ $\alpha \sigma\left[s_{1}^{\prime}, s_{2}^{\prime}\right]_{\left(u_{1}, u_{2}\right)}$ and $\left(\alpha \sigma\left[s_{1}^{\prime}\right]_{u_{1}}\right)_{\mid u_{2}}=\alpha \sigma_{\mid u_{2}}$. Thus, $s \theta \stackrel{\geq U}{\rightarrow}{ }^{*}$ $\alpha \sigma\left[s_{1}^{\prime}, s_{2}^{\prime}\right]_{\left(u_{1}, u_{2}\right)} \stackrel{\geq\left\{u_{1}, u_{2}\right\}}{\rightarrow} \alpha \sigma$. By repeating similar arguments to the above, there exist $s_{1}^{\prime}, \cdots, s_{n}^{\prime}$ such that $s \theta \stackrel{\geq U}{\rightarrow} \alpha\left[s_{1}^{\prime}, \cdots, s_{n}^{\prime}\right]_{\left(u_{1}, \cdots, u_{n}\right)} \stackrel{\geq O_{X}\left(\alpha^{\prime}\right)}{\rightarrow^{*}} \alpha \sigma$ where for each $i \in\{1, \cdots, n\}, s_{i}^{\prime}=s_{\mid u_{i}} \theta, s_{i}^{\prime} \in \operatorname{Bud}\left(R_{\mathrm{C}}\right)$, or $s_{i}^{\prime}=x \theta_{\mid v}$ for some $x \in \mathrm{V}(s), v \in O(x \theta)$. Let $\rho^{\prime}=\left\{\alpha_{\mid u_{i}}^{\prime} \rightarrow s_{i}^{\prime} \mid 1 \leq\right.$ $i \leq n\}$. Then, $\rho^{\prime} \in \operatorname{BudMap}_{R}(s \theta, \alpha)$. We define $\rho$ as follows: $y \rho=y$ if $y \rho \in\left\{x \theta_{\mid v} \mid x \in \mathrm{V}(s), v \in O(x \theta)\right\}$, $y \rho=s_{\mid u_{i}}$ if $y \rho^{\prime}=s_{\mid u_{i}} \theta$ and $y=\alpha^{\prime} \mid u_{i}, y \rho=y \rho^{\prime}$ otherwise. Then, $\rho \in \overline{\operatorname{BudMap}}_{R}(s, \alpha)$ and $\rho^{\prime}=\rho \theta^{\prime}$ hold for substitution $\theta^{\prime}: \mathrm{V}(s) \cup \mathrm{V}\left(\alpha^{\prime}\right) \rightarrow \operatorname{Psub}(s \theta)$ satisfying that if $x \in \mathrm{V}(s)$ then $x \theta^{\prime}=x \theta$, otherwise if $x=\alpha_{u_{i}}^{\prime}$ then $x \theta^{\prime}=s_{i}^{\prime}$. Note that if $s_{i}^{\prime}=x \theta_{\mid v}$ for some $x \in \mathrm{V}(s), v \in O(x \theta)$, then $s_{i}^{\prime}$ is minimum since $x \theta$ is minimum by Lemma 4.2. Hence, $\theta^{\prime}$ is a locally minimum substitution. Thus, (2) of this lemma holds.

\section{R-Unification Algorithm}

We now give our $R$-unification algorithm for confluent semiconstructor TRSs which is based on the unification algorithm in [14] applicable to confluent right-ground TRSs. The algorithm in [14] is constructed by using algorithms of deciding joinability and reachability for right-ground TRSs, but only joinability is decidable for confluent semiconstructor TRSs [10]. (Undecidability of the reachability has been shown in [11].) Thus, our unification algorithm can be considered as a refined version of that of [14] in 
the sense that no algorithm of deciding reachability of semiconstructor TRSs is needed, (though a decision algorithm of reachability for right-ground TRSs is used) and some primitive operations are unified or simplified.

Each primitive operation $\Phi$ of our algorithm takes a finite set of pairs $\Gamma \subseteq E_{0}$ and produces some $\tilde{\Gamma} \subseteq E_{0}$, denoted by $\Gamma \Rightarrow_{\Phi} \tilde{\Gamma}$. This operation is called a transformation. Such a transformation is made nondeterministically: $\Gamma \Rightarrow_{\Phi} \Gamma_{1}, \Gamma \Rightarrow_{\Phi} \Gamma_{2}, \cdots, \Gamma \Rightarrow_{\Phi} \Gamma_{k}$ are allowed for some $\Gamma_{1}, \cdots, \Gamma_{k} \subseteq E_{0}$. In this case, we write $\Phi(\Gamma)=\left\{\Gamma_{1}, \cdots, \Gamma_{k}\right\}$ regarding $\Phi$ as a function. Let $\Rightarrow_{\Phi}^{*}$ be the reflexive transitive closure of $\Rightarrow_{\Phi}$. Our algorithm starts from $\Gamma_{0}=\left\{s_{0} \approx t_{0}\right\}$ and makes primitive transformations repeatedly. We will prove that there exists a sequence $\Gamma_{0} \Rightarrow_{\Phi}^{*} \Gamma$ such that $\Gamma$ is $\emptyset$-unifiable iff $\Gamma_{0}$ is $R$-unifiable.

Our algorithm is divided into three stages. Stage I repeatedly decomposes a set of term pairs $\Gamma$ into another one $\tilde{\Gamma}$ by guessing a rewrite rule applied at the root position of a non-variable subterm of some term appearing in $\Gamma$. Finally, Stage I transforms $\Gamma$ into a set of type vf pairs $\Gamma_{\mathrm{f}}$, which becomes an input of the next Stage II. Stage II is similar to a usual $\emptyset$-unification algorithm and stops when a set of type vf pairs $\Gamma$ is in solved form as explained later. The Final Stage only checks $\emptyset$-unifiability of $\Gamma$ in solved form. rithm.

We give the definition related to validity of the algo-

Definition 5.1: Substitutions $\theta$ and $\theta^{\prime}$ are consistent if $x \theta=x \theta^{\prime}$ for any $x \in \operatorname{Dom}(\theta) \cap \operatorname{Dom}\left(\theta^{\prime}\right)$.

Definition 5.2: [14] Let $\Phi: \mathcal{P}\left(E_{0}\right) \rightarrow \mathcal{P}\left(\mathcal{P}\left(E_{0}\right)\right)$ be a transformation. Then, $\Phi$ is valid if the following validity conditions (V1) and (V2) hold. For any $\Gamma \subseteq E_{0}$, let $\Phi(\Gamma)=$ $\left\{\Gamma_{1}, \cdots, \Gamma_{n}\right\}$.

(V1) If $\theta$ is a locally minimum $R$-unifier of $\Gamma$, then there exists $i \in\{1, \cdots, n\}$ and a substitution $\theta^{\prime}$ such that $\theta^{\prime}$ is consistent with $\theta$ and $\theta^{\prime}$ is a locally minimum $R$ unifier of $\Gamma_{i}$.

(V2) If there exists $i \in\{1, \cdots, n\}$ such that $\operatorname{Core}\left(\Gamma_{i}\right)$ is $R$ unifiable, then $\operatorname{Core}(\Gamma)$ is $R$-unifiable.

\section{$5.1 \quad$ Stage I}

The transformation $\Phi_{1}$ of Stage I takes as input a finite subset of pairs $\Gamma \subseteq E_{0}$ and has a finite number of nondeterministic choices $\Gamma \Rightarrow_{\Phi_{1}} \Gamma_{1}, \cdots, \Gamma \Rightarrow_{\Phi_{1}} \Gamma_{k}$ for some $\Gamma_{1}, \cdots, \Gamma_{k} \subseteq E_{0}$. We consider all possibilities in order to ensure the correctness of the algorithm.

We begin with the initial $\Gamma=\left\{s_{0} \approx t_{0}\right\}$ and repeatedly apply the transformation $\Phi_{1}$ until the current $\Gamma$ becomes a set of type vf pairs with or without fail. This condition is called the stop condition of Stage I and defined as $\Gamma \subseteq\left\{\right.$ fail, $\left.s \approx_{\mathrm{vf}} t \mid s, t \in T\right\}$. If $\Gamma$ satisfies this condition, then $\Gamma$ becomes an input of the next stage.

To describe the transformations used in Stage I, we need the following auxiliary function.

$$
\begin{aligned}
& \operatorname{Dec}\left(f\left(s_{1}, \cdots, s_{n}\right), f\left(t_{1}, \cdots, t_{n}\right)\right) \\
& =\left\{s_{i} \triangleright t_{i} \mid 1 \leq i \leq n, t_{i} \in S\right\} \\
& \cup\left\{s_{i} \approx t_{i} \mid 1 \leq i \leq n, s_{i} \notin G, t_{i} \notin S\right\} \\
& \cup\left\{t_{i} \approx_{\mathrm{vf}} s_{i} \mid 1 \leq i \leq n, s_{i} \in G, t_{i} \notin S\right\}
\end{aligned}
$$

In Stage I, we nondeterministically apply Conversion or choose an element $p$ in $\Gamma \backslash(G \cup X) \times(G \cup X)$ and apply one of the following transformations (TT, VT) to $\Gamma$ according to form of the chosen $p=s \approx t$ or $s \triangleright t$.

If no transformation is possible, $\Gamma \Rightarrow_{\Phi_{1}}$ \{fail $\}$. We write $s \simeq t$ if $s \approx t$ or $t \approx s$. We say that $p=s \simeq t$ satisfies the TT condition if $s, t \notin X$ and either $s \notin G$ or $t \notin G$, and the VT condition if $s \in X$ and $t \in S$. Similarly, we say that $p=s \triangleright t$ satisfies the TT condition if $s \notin X$, and the VT condition if $s \in X$. Note that if $p=s \triangleright t$ then $t \in S$.

Let $\Gamma^{\prime}=\Gamma \backslash\{p\}$. In the following explanations, we assume that $\theta$ is a locally minimum unifier of $p$ and we list the conditions that are assumed on a proof $\gamma$ of $p$. When applying the transformations we of course lack this information and so we just have to check that the conditions of the transformations are satisfied.

\subsubsection{Conversion}

If every $s \approx t, s \triangleright t \in \Gamma$ does not satisfy the TT condition, then

$$
\Gamma \Rightarrow_{\Phi_{1}} \operatorname{Conv}(\Gamma)
$$

where $\operatorname{Conv}(\Gamma)=\left\{x \approx_{\mathrm{vf}} s \mid x \approx s \in \Gamma\right.$ or $s \approx x \in \Gamma$ or $x \triangleright s \in$ $\Gamma$ or $\left.x \approx_{\mathrm{vf}} s \in \Gamma\right\} \cup\left\{s \approx_{\mathrm{vf}} t \in G \times G \mid s \approx t \in \Gamma\right.$ or $s \approx_{\mathrm{vf}} t \in$ $\Gamma\} \cup\{$ fail $\mid$ fail $\in \Gamma\}$. Note that $\operatorname{Conv}(\Gamma)$ satisfies the stop condition of Stage I.

In the following examples, we use the TRS $\mathrm{M}\left(R_{\mathrm{e}}\right)$ of Example Appendix B.3.

\section{Example 5.3:}

$$
\begin{aligned}
& \left\{x \approx x, \mathrm{t} \approx_{\mathrm{vf}} \mathrm{t}, x \approx \mathrm{t}, x \approx x^{\prime}, \mathrm{t} \approx x\right\} \\
& \Rightarrow_{\Phi_{1}}\left\{x \approx_{\mathrm{vf}} x, \mathrm{t} \approx_{\mathrm{vf}} \mathrm{t}, x \approx_{\mathrm{vf}} \mathrm{t}, x \approx_{\mathrm{vf}} x^{\prime}, x \approx_{\mathrm{vf}} \mathrm{t}\right\} \\
& \{x \approx \operatorname{nand}(\mathrm{t}, x), x \approx \mathrm{f}, \mathrm{f} \approx \mathrm{t}\} \\
& \Rightarrow_{\Phi_{1}}\left\{x \approx_{\mathrm{vf}} \operatorname{nand}(\mathrm{t}, x), x \approx_{\mathrm{vf}} \mathrm{f}, \mathrm{f} \approx_{\mathrm{vf}} \mathrm{t}\right\}
\end{aligned}
$$

\section{TT Transformation}

1. If $p=s \simeq t$ satisfies the TT condition, we choose one of the following three cases. Let $k=\operatorname{ar}(\operatorname{root}(s))$. We guess that $\theta$ is a locally minimum $R$-unifier of $p$ and that there exists a joinable sequence $\gamma: s \theta \downarrow t \theta$.

a. If $\operatorname{root}(s)=\operatorname{root}(t)$, then

$$
\Gamma^{\prime} \cup\{p\} \Rightarrow_{\Phi_{1}} \Gamma^{\prime} \cup\left\{s_{\mid i} \approx t_{\mid i} \mid 1 \leq i \leq k\right\}
$$

In this transformation, we guess that $\gamma: s \theta \downarrow t \theta$ is $\varepsilon$-invariant. 
b. If $s \notin G$, then we choose a fresh variant of a rule $\alpha \rightarrow \beta \in R$ that satisfies $\operatorname{root}(s)=\operatorname{root}(\alpha)$ and

$$
\Gamma^{\prime} \cup\{p\} \Rightarrow_{\Phi_{1}} \Gamma^{\prime} \cup \operatorname{Dec}(s, \alpha) \cup\{\beta \approx t\}
$$

In this transformation, we guess that $\alpha \sigma \rightarrow \beta \sigma$ is the leftmost $\varepsilon$-reduction step in $\gamma: s \theta \rightarrow^{*} \alpha \sigma \rightarrow$ $\beta \sigma \downarrow t \theta$ for some substitution $\sigma$ (where the subsequence $s \theta \rightarrow^{*} \alpha \sigma$ is $\varepsilon$-invariant).

c. If $s \in G$, then we choose a term $s^{\prime} \in \operatorname{Aux}(s)$ and

i. If $\operatorname{root}\left(s^{\prime}\right)=\operatorname{root}(t)$,

$$
\Gamma^{\prime} \cup\{p\} \Rightarrow_{\Phi_{1}} \Gamma^{\prime} \cup\left\{s_{\mid i}^{\prime} \approx t_{\mid i} \mid 1 \leq i \leq k\right\}
$$

ii. We choose a rule $\alpha \rightarrow \beta \in R_{\mathrm{rg}}$ that satisfies $s^{\prime} \rightarrow_{R_{\mathrm{rg}}}^{+} \beta$ and

$$
\Gamma^{\prime} \cup\{p\} \Rightarrow_{\Phi_{1}} \Gamma^{\prime} \cup\{\beta \approx t\}
$$

and then do a single TT transformation on $t \approx \beta$ as in 1.a or 1.b.

In this transformation, we guess an auxiliary term $s^{\prime}$ satisfying that $s \downarrow s^{\prime} \rightarrow_{R_{\mathrm{rg}}}^{*} \leftarrow^{*} t \theta$ by Lemma 3.10. Moreover, we guess that $\gamma^{\prime}$ : $s^{\prime} \rightarrow_{R_{\mathrm{rg}}}^{*} \leftarrow^{*} t \theta$ is $\varepsilon$-invariant or $\alpha \sigma \rightarrow \beta$ is the rightmost $\varepsilon$-reduction in the subsequence $s^{\prime} \rightarrow_{R_{\mathrm{rg}}}^{*} r$ of $\gamma^{\prime}: s^{\prime} \rightarrow_{R_{\mathrm{rg}}}^{*} r \leftarrow^{*} t \theta$.

2. If $p=s \triangleright t$ satisfies the TT condition, we choose one of the following three cases. We guess that there exists a sequence $\gamma: s \theta \rightarrow^{*} r \stackrel{\geq O_{X}(t)}{\leftrightarrow} t \theta$ for some term $r$.

a. If $\operatorname{root}(s)=\operatorname{root}(t)$, then

$$
\Gamma^{\prime} \cup\{p\} \Rightarrow_{\Phi_{1}} \Gamma^{\prime} \cup \operatorname{Dec}(s, t)
$$

In this transformation, we guess that $\gamma: s \theta \rightarrow^{*}$ $r \stackrel{\geq O_{X}(t)}{\leftrightarrow}{ }^{*} t \theta$ is $\varepsilon$-invariant.

b. If $s \notin G$, we choose a position $v \in O(s)$ such that $s_{\mid v} \in S$, and terms $s_{1} \cdots s_{n}$ where $\operatorname{Min}\left(O_{G}\left(s_{\mid v}\right)\right)=$ $\left\{u_{1}, \cdots, u_{n}\right\}$ and $s_{i} \in \operatorname{Aux}\left(s_{\mid v u_{i}}\right)$ for $i \in\{1 \cdots n\}$. Let $s^{\prime}=s_{\mid v}\left[s_{1} \cdots, s_{n}\right]_{\left(u_{1}, \cdots, u_{n}\right)}$. Then, we choose a fresh variant of a rule $\alpha \rightarrow \beta \in R$ with $\operatorname{root}\left(s^{\prime}\right)=$ $\operatorname{root}(\alpha)$, a substitution $\rho \in \overline{\operatorname{BudMap}}_{R}\left(s^{\prime}, \alpha\right)$, and

$$
\begin{aligned}
& \Gamma^{\prime} \cup\{p\} \\
& \Rightarrow \Phi_{\Phi_{1}} \Gamma^{\prime} \cup \operatorname{Dec}\left(s^{\prime}, \alpha^{\prime} \rho\right) \\
& \cup\left\{x \rho \approx x^{\prime} \rho \mid x \in \mathrm{V}(\alpha), x \equiv x^{\prime}\right\} \\
& \cup\left\{s[\beta \rho]_{v} \triangleright t\right\}
\end{aligned}
$$

Here, $\alpha^{\prime}=$ linearize $(\alpha)$. In this transformation, we guess the sequence $\gamma^{\prime}: s\left[s^{\prime}\right]_{v} \theta \rightarrow^{*} s \theta[\alpha \sigma]_{v} \rightarrow$ $s \theta[\beta \sigma]_{v} \rightarrow^{*} \quad r \stackrel{\geq O_{X}(t)}{\leftrightarrow} \quad t \theta$ for some $\sigma$ where $s \theta[\alpha \sigma]_{v} \rightarrow s \theta[\beta \sigma]_{v}$ is the first reduction at non-ground and non-variable position of $s$, i.e., the subsequence $s\left[s^{\prime}\right]_{v} \theta \rightarrow^{*} s \theta[\alpha \sigma]_{v}$ is $O_{X}(s)$ frontier. By Lemma 4.12(2), there exist a substitution $\rho \in \overline{\operatorname{BudMap}}_{R}\left(s^{\prime}, \alpha\right)$ and a locally minimum substitution $\theta^{\prime}$ such that $s^{\prime} \theta \stackrel{\geq O_{X}\left(s^{\prime}\right) \cup \operatorname{Min}\left(O_{G}\left(s^{\prime}\right)\right)}{\rightarrow}$ $\alpha^{\prime} \rho \theta^{\prime} \stackrel{\geq O_{X}\left(\alpha^{\prime}\right)}{\rightarrow} \alpha \sigma$ and $\beta \rho \theta^{\prime} \stackrel{\geq O_{X}(\beta)}{\rightarrow} \beta \sigma$.

c. If $s \in G$, then we choose a term $s^{\prime} \in \operatorname{Aux}(s)$ and

i. If $\operatorname{root}\left(s^{\prime}\right)=\operatorname{root}(t)$,

$$
\Gamma^{\prime} \cup\{p\} \Rightarrow_{\Phi_{1}} \Gamma^{\prime} \cup \operatorname{Dec}\left(s^{\prime}, t\right)
$$

ii. We choose a rule $\alpha \rightarrow \beta \in R_{\mathrm{rg}}$ that satisfies $s^{\prime} \rightarrow_{R_{\mathrm{rg}}}^{+} \beta$ and $\operatorname{root}(\beta)=\operatorname{root}(t)$, and

$$
\Gamma^{\prime} \cup\{p\} \Rightarrow_{\Phi_{1}} \Gamma^{\prime} \cup\{\beta \triangleright t\}
$$

Then, we do a single TT transformation on $\beta \triangleright t$ as in 2.a.

In this transformation, we guess that $\alpha \sigma \rightarrow$ $\beta$ is the rightmost $\varepsilon$-reduction step in $\gamma: s^{\prime} \rightarrow_{R_{\mathrm{rg}}}^{*} \alpha \sigma \rightarrow \beta \rightarrow^{*} r \stackrel{\geq O_{X}(t)}{\leftrightarrow}{ }^{*} t \theta$ for some substitution $\sigma$. Thus, the subsequence $\gamma^{\prime}($ of $\gamma): \beta \rightarrow^{*} r \stackrel{\geq O_{X}(t)}{\leftrightarrow} t \theta$ is $\varepsilon$-invariant. This ensures that case 2.a of the TT transformation is applicable to $\beta \triangleright t$.

\section{Example 5.4:}

(1) By choosing auxiliary term $t \in \operatorname{Aux}(\mathrm{t})$ and rule $t \rightarrow$ nand $(f, f)$ and applying case $1 . c$, we get

$$
\begin{aligned}
& \Gamma^{\prime} \cup\{\mathrm{t} \approx \operatorname{nand}(x, \mathrm{t})\} \\
& \Rightarrow_{\Phi_{1}} \Gamma^{\prime} \cup\{\operatorname{nand}(\mathrm{f}, \mathrm{f}) \approx \operatorname{nand}(x, \mathrm{t})\}
\end{aligned}
$$

Then, we apply case $1 . \mathrm{a}$ of the TT transformation to $\operatorname{nand}(x, \mathrm{t}) \approx \operatorname{nand}(\mathrm{t}, \mathrm{t})$ and get

$$
\Gamma^{\prime} \cup\{x \approx \mathrm{f}, \mathrm{f} \approx \mathrm{t}\}
$$

(2) By applying case 2.a repeatedly, we get

$$
\begin{aligned}
& \Gamma^{\prime} \cup\left\{\neg\left(\wedge\left(x^{\prime \prime}, x^{\prime \prime}\right)\right) \triangleright \neg\left(\wedge\left(x^{\prime}, x^{\prime}\right)\right)\right\} \\
& \Rightarrow_{\Phi_{1}} \Gamma^{\prime} \cup\left\{\wedge\left(x^{\prime \prime}, x^{\prime \prime}\right) \triangleright \wedge\left(x^{\prime}, x^{\prime}\right)\right\} \\
& \Rightarrow_{\Phi_{1}} \Gamma^{\prime} \cup\left\{x^{\prime \prime} \approx x^{\prime}\right\}
\end{aligned}
$$

(3) Let $p$ be nand $(x, \mathrm{t}) \triangleright \neg\left(\wedge\left(x^{\prime}, x^{\prime}\right)\right)$. We apply case 2.b. First, we choose $v=\varepsilon$. Here, $\operatorname{Min}\left(O_{G}(\operatorname{nand}(x, \mathrm{t}))\right)=$ $\{2\}$, so we choose auxiliary term $\mathrm{t}$ as $s_{1}$. Next, we choose rule nand $\left(x^{\prime \prime}, x^{\prime \prime}\right) \rightarrow \neg\left(\wedge\left(x^{\prime \prime}, x^{\prime \prime}\right)\right)$. Let linearize $\left(\operatorname{nand}\left(x^{\prime \prime}, x^{\prime \prime}\right)\right)$ be nand $\left(x^{\prime \prime}, x_{1}^{\prime \prime}\right)$, so that $x^{\prime \prime} \equiv$ $x_{1}^{\prime \prime}$. Moreover, we choose $\rho=\left\{x^{\prime \prime} \rightarrow x^{\prime \prime}, x_{1}^{\prime \prime} \rightarrow \mathrm{t}\right\}$, and we get

$$
\begin{aligned}
& \Gamma^{\prime} \cup\left\{\operatorname{nand}(x, \mathrm{t}) \triangleright \neg\left(\wedge\left(x^{\prime}, x^{\prime}\right)\right)\right\} \\
& \Rightarrow_{\Phi_{1}} \Gamma^{\prime} \cup \operatorname{Dec}\left(\operatorname{nand}(x, \mathrm{t}), \operatorname{nand}\left(x^{\prime \prime}, \mathrm{t}\right)\right) \\
& \cup\left\{x^{\prime \prime} \approx \mathrm{t}\right\} \cup\left\{\neg\left(\wedge\left(x^{\prime \prime}, x^{\prime \prime}\right)\right) \triangleright \neg\left(\wedge\left(x^{\prime}, x^{\prime}\right)\right)\right\} \\
& =\Gamma^{\prime} \cup\left\{x \approx x^{\prime \prime}, \mathrm{t} \approx_{\mathrm{vf}} \mathrm{t}, x^{\prime \prime} \approx \mathrm{t},\right. \\
& \left.\neg\left(\wedge\left(x^{\prime \prime}, x^{\prime \prime}\right)\right) \triangleright \neg\left(\wedge\left(x^{\prime}, x^{\prime}\right)\right)\right\}
\end{aligned}
$$


VT transformation

1. If $p=x \simeq s$ satisfies the VT condition, we choose a position $v \in O(s)$ such that $s_{\mid v} \in S$ and apply one of the following two cases.

a. We choose a fresh variant of a rule $\alpha \rightarrow \beta \in R$ that satisfies $\operatorname{root}\left(s_{\mid v}\right)=\operatorname{root}(\alpha)$ and

$$
\Gamma^{\prime} \cup\{p\} \Rightarrow_{\Phi_{1}} \Gamma^{\prime} \cup \operatorname{Dec}\left(s_{\mid v}, \alpha\right) \cup\left\{s[\beta]_{v} \approx x\right\}
$$

In this transformation, we guess that $s \theta[\alpha \sigma]_{v} \rightarrow$ $s \theta[\beta \sigma]_{v}$ is the leftmost $v$-reduction step in $\gamma: s \theta \rightarrow^{*} s \theta[\alpha \sigma]_{v} \rightarrow s \theta[\beta \sigma]_{v} \downarrow x \theta$ for some $\sigma$ and $v \in \operatorname{Min}(\mathcal{R}(\gamma)$ ) (where the subsequence $s \theta \rightarrow^{*} s \theta[\alpha \sigma]_{v}$ is $v$-invariant).

b. We choose a constant $c$ and

$$
\Gamma^{\prime} \cup\{p\} \Rightarrow_{\Phi_{1}} \Gamma^{\prime} \cup\left\{x \approx s[c]_{v}, c \approx s_{\mid v}\right\}
$$

where if $s[c]_{v} \in G$, then $x \approx s[c]_{v}$ is replaced by $x \approx_{\mathrm{vf}} s[c]_{v}$. In this transformation, we guess that there exists $\gamma: x \theta \rightarrow^{*} r \leftarrow^{*} s \theta$ for some $r$, and $v \in \operatorname{Min}(\mathcal{R}(\gamma)) \cap \operatorname{Min}\left(\mathcal{R}\left(\gamma^{\prime}\right)\right)$ where $\gamma^{\prime}: x \theta \rightarrow^{*}$ $r$ is the subsequence of $\gamma$. Note that since $x \theta$ is minimum, only leaf symbols of $x \theta$ are rewritten in $\gamma^{\prime}$ by Lemma 4.3. That is, we guess $x \theta_{\mid v}=c$ and $c \downarrow s \theta_{\mid v}$.

2. If $p=x \triangleright s$ satisfies the VT condition, we choose a constant $c$ and a position $v \in O(s)$ such that $\left.s\right|_{v} \in S$. Then

$$
\Gamma^{\prime} \cup\{p\} \Rightarrow_{\Phi_{1}} \Gamma^{\prime} \cup\left\{x \triangleright s[c]_{v}, c \triangleright s_{\mid v}\right\}
$$

If $s[c]_{v} \in G$, then $x \triangleright s[c]_{v}$ is replaced by $x \approx_{\mathrm{vf}} s[c]_{v}$. In this transformation, we guess that there exists $\gamma$ :

$x \theta \rightarrow^{*} r \stackrel{\geq O_{X}(s)}{\leftrightarrow}{ }^{*} s \theta$ for some $r$, and $v \in \operatorname{Min}(\mathcal{R}(\gamma)) \cap$ $\operatorname{Min}\left(\mathcal{R}\left(\gamma^{\prime}\right)\right)$ where $\gamma^{\prime}: x \theta \rightarrow^{*} r$. Note that since $x \theta$ is minimum, only leaf symbols of $x \theta$ are rewritten in $\gamma^{\prime}$ by Lemma 4.3. That is, we guess $x \theta_{\mid v}=c$ and $c \rightarrow^{*}$ $r_{\mid v} \stackrel{\geq O_{X}\left(s_{\mid v}\right)}{\leftrightarrow}{ }^{*} s \theta_{\mid v}$.

\section{Example 5.5:}

(1) By choosing $v=\varepsilon$ and rule $\operatorname{nand}\left(\neg\left(\wedge\left(x^{\prime}, x^{\prime}\right)\right), x^{\prime}\right) \rightarrow \mathrm{t}$ and applying case 1 .a, we get

$$
\begin{aligned}
& \Gamma^{\prime} \cup\{\operatorname{nand}(\operatorname{nand}(x, \mathrm{t}), x) \approx x\} \\
& \Rightarrow \Phi_{1} \Gamma^{\prime} \cup \operatorname{Dec}(\operatorname{nand}(\operatorname{nand}(x, \mathrm{t}), x), \\
& \left.\operatorname{nand}\left(\neg\left(\wedge\left(x^{\prime}, x^{\prime}\right)\right), x^{\prime}\right)\right) \cup\{\mathrm{t} \approx x\} \\
& =\Gamma^{\prime} \cup\left\{\operatorname{nand}(x, \mathrm{t}) \triangleright \neg\left(\wedge\left(x^{\prime}, x^{\prime}\right)\right), x \approx x^{\prime},\right. \\
& \mathrm{t} \approx x\}
\end{aligned}
$$

(2) By choosing $v=1$ and constant t and applying case 1.b, we get

$$
\begin{aligned}
& \Gamma^{\prime} \cup\{\operatorname{nand}(\operatorname{nand}(x, \mathrm{t}), x) \approx x\} \\
& \Rightarrow_{\Phi_{1}} \Gamma^{\prime} \cup\{x \approx \operatorname{nand}(\mathrm{t}, x), \mathrm{t} \approx \operatorname{nand}(x, \mathrm{t})\}
\end{aligned}
$$

\subsection{Stage II}

Below we define the one step transformation $\Phi_{2}$ of Stage II. We write $\Gamma \Rightarrow_{\Phi_{2}} \tilde{\Gamma}$ if $\Phi_{2}(\Gamma) \ni \tilde{\Gamma}$.

We begin with $\Gamma$ which is $\{$ fail $\}$ or produced by Conversion of Stage I. Hence, $\Gamma \subseteq$ \{fail, $s \approx_{\mathrm{vf}} t \mid(s, t) \in$ $(X \times T) \cup(G \times G)\}$ holds. Then, we repeatedly apply the transformation $\Phi_{2}$ until the current $\Gamma$ satisfies the stop condition of Stage II defined below. In Stage II, any pair $s \approx_{\mathrm{vf}} t$ in $\Gamma$ satisfies $s \notin S$. We consider all possibilities in order to ensure the correctness of the algorithm. If $\Gamma$ satisfies the stop condition, then we check the $\emptyset$-unifiability of $\Gamma$ in the Final Stage.

Definition 5.6: $\quad \Gamma$ is in solved form if for any $x \approx_{\mathrm{vf}} s$ and $x \approx_{\mathrm{vf}} t$ in $\Gamma, s, t \notin X$ and $s=t$ hold.

The stop condition of Stage II is that $\Gamma$ satisfies one of the following two conditions.

(1) For any $s \approx_{\mathrm{vf}} t \in \Gamma$, we have $s \in X$ and $\Gamma$ is in solved form.

(2) $\Gamma=\{$ fail $\}$.

(Note. $\Gamma=\emptyset$ satisfies condition (1).)

\section{Definition 5.7:}

(1) For any $t$ and set of pairwise disjoint positions $U$, $\operatorname{gmin}(t, U)=t\left[t_{1}, \cdots, t_{n}\right]_{\left(u_{1}, \cdots, u_{n}\right)}$, where $U \cap O_{G}(t)=$ $\left\{u_{1}, \cdots, u_{n}\right\}$ and $t_{i}$ be the minimum term in $\mathcal{L}\left(t_{\mid u_{i}}\right)$ for $i \in\{1, \cdots, n\}$. Note that $O_{X}(t)=O_{X}(\operatorname{gmin}(t, U))$ and $\operatorname{Min}\left(O_{G}(t)\right)=\operatorname{Min}\left(O_{G}(\operatorname{gmin}(t, U))\right)$.

(2) For $s, t \notin X$, we define predicate $\operatorname{common}(s, t)$ as follows. Predicate common $(s, t)$ is true if $O(s) \cap$ $O(t) \supseteq \operatorname{Min}\left(O_{X}(s) \cup O_{X}(t)\right)$ and $s[c, \cdots, c]_{\left(u_{1}, \cdots, u_{n}\right)}=$ $t[c, \cdots, c]_{\left(u_{1}, \cdots, u_{n}\right)}$, where $\operatorname{Min}\left(O_{X}(s) \cup O_{X}(t)\right)=$ $\left\{u_{1}, \cdots, u_{n}\right\}$. For example, let $s=f\left(s^{\prime}, x, s^{\prime \prime}\right)$ and $t=f\left(s^{\prime}, t^{\prime \prime}, y\right)$, where $s^{\prime} \in G$. In this example, $\operatorname{Min}\left(O_{X}(s) \cup O_{X}(t)\right)=\{2,3\}$. Since $s[c, c]_{(2,3)}=$ $f\left(s^{\prime}, c, c\right)=t[c, c]_{(2,3)}$, common $(s, t)$ holds.

In Stage II, we first choose an element $p$ in $\Gamma$ nondeterministically and then apply one of the following transformations to $\Gamma$ according to the type of the chosen $p$. If $\Gamma$ does not satisfy the stop condition of Stage II and no transformation is possible, $\Gamma \Rightarrow_{\Phi_{2}}$ \{fail $\}$. Let $\Gamma^{\prime}=\Gamma \backslash\{p\}$.

Decomposition

If $p=x \approx_{\mathrm{vf}} s$ with $s \in S$ and there exists a pair $q=x \approx_{\mathrm{vf}} t \in$ $\Gamma$ such that $s \neq t$ and $t \in S$, and common $\left(s^{\prime}, t^{\prime}\right)$, where $s^{\prime}=$ $\operatorname{gmin}(s, U \cup V), t^{\prime}=\operatorname{gmin}(t, U \cup V), U=\operatorname{Min}\left(O_{X}(s) \cup O_{X}(t)\right)$, $V=\operatorname{Min}\left(O_{G}(s) \cap O_{G}(t)\right)$, then

$$
\begin{aligned}
& \Gamma^{\prime \prime} \cup\{p, q\} \\
& \Rightarrow_{\Phi_{2}} \Gamma^{\prime \prime} \cup\left\{q^{\prime}\right\} \\
& \cup\left\{s_{\mid u}^{\prime} \approx_{\mathrm{vf}} t_{\mid u}^{\prime} \mid u \in U \text { and } s_{\mid u}^{\prime} \in X\right\} \\
& \cup\left\{t_{\mid u}^{\prime} \approx_{\mathrm{vf}} s_{\mid u}^{\prime} \mid u \in U \text { and } s_{\mid u}^{\prime} \notin X\right\}
\end{aligned}
$$


where $\Gamma^{\prime \prime}=\Gamma^{\prime} \backslash\{q\}$ and $q^{\prime}=x \approx_{\mathrm{vf}} t^{\prime}$. Here, we assume that $s^{\prime} \gg_{\mathrm{HD}_{G}} t^{\prime}$.

Example 5.8: $\quad$ Let $\Gamma=\{p, q\}$ with $p=$ $x \approx_{\mathrm{vf}} \operatorname{nand}(\operatorname{nand}(\neg(y)$, nand $(\mathrm{f}, \mathrm{f})), \mathrm{t})$ and $q=$ $x \approx_{\mathrm{vf}} \operatorname{nand}(\operatorname{nand}(x, \mathrm{t}), x)$. Then, $p^{\prime}=\operatorname{nand}(\operatorname{nand}(\neg(y), \mathrm{t}), \mathrm{t})$ and $q^{\prime}=q$, and common (nand $(\operatorname{nand}(\neg(y), \mathrm{t}), \mathrm{t})$, $\operatorname{nand}(\operatorname{nand}(x, \mathrm{t}), x))$, because $\operatorname{nand}(\operatorname{nand}(\neg(y), \mathrm{t}), \mathrm{t})[c, c]_{(11,2)}=\operatorname{nand}(\operatorname{nand}(c, \mathrm{t}), c)=$ $\operatorname{nand}(\operatorname{nand}(x, \mathrm{t}), x)[c, c]_{(11,2)}$ holds. Moreover, $\mathrm{HD}_{G}(\operatorname{nand}(\operatorname{nand}(\neg(y), \mathrm{t}), \mathrm{t}))=\{0,1,6,11\}_{\mathrm{m}} \gg\{0,0,5,10\}_{\mathrm{m}}$ $=\mathrm{HD}_{G}(\operatorname{nand}(\operatorname{nand}(x, \mathrm{t}), x))$ holds. So, we can make the following Decomposition:

$$
\{p, q\} \Rightarrow_{\Phi_{2}}\left\{q^{\prime}, x \approx_{\mathrm{vf}} \neg(y), x \approx_{\mathrm{vf}} \mathrm{t}\right\}
$$

Substitution

If $p=x \approx_{\mathrm{vf}} s$ or $s \approx_{\mathrm{vf}} x$ with $s \notin S$, then

$$
\Gamma^{\prime} \cup\{p\} \Rightarrow_{\Phi_{2}} \Gamma^{\prime} \sigma
$$

where $\sigma=\left\{x \rightarrow s^{\prime}\right\}$ and $s^{\prime}$ is the minimum term in $\mathcal{L}(s)$. Note that if $s \in X$ then $s$ is the minimum term in $\mathcal{L}(s)$.

\section{Example 5.9:}

$$
\begin{aligned}
& \left\{\mathrm{t} \approx_{\mathrm{vf}} \mathrm{t}, x^{\prime \prime} \approx_{\mathrm{vf}} \mathrm{t}, x^{\prime \prime} \approx_{\mathrm{vf}} x^{\prime}, x \approx_{\mathrm{vf}} x^{\prime}, x \approx_{\mathrm{vf}} \mathrm{t}\right\} \\
& \cup\left\{x \approx_{\mathrm{vf}} x^{\prime \prime}\right\} \Rightarrow_{\Phi_{2}}\left\{\mathrm{t} \approx_{\mathrm{vf}} \mathrm{t}, x^{\prime \prime} \approx_{\mathrm{vf}} \mathrm{t}, x^{\prime \prime} \approx_{\mathrm{vf}} x^{\prime}\right\} \\
& \left\{\mathrm{t} \approx_{\mathrm{vf}} \mathrm{t}, x^{\prime \prime} \approx_{\mathrm{vf}} x^{\prime}\right\} \cup\left\{x^{\prime \prime} \approx_{\mathrm{vf}} \mathrm{t}\right\} \Rightarrow_{\Phi_{2}} \\
& \left\{\mathrm{t} \approx_{\mathrm{vf}} \mathrm{t}, \mathrm{t} \approx_{\mathrm{vf}} x^{\prime}\right\}
\end{aligned}
$$

\section{GT Transformation}

If $p=s \approx_{\mathrm{vf}} t$ with $s \in G, t \notin X$, and common $\left(s^{\prime}, t^{\prime}\right)$ where $s^{\prime}=\operatorname{gmin}(s, U \cup V), t^{\prime}=\operatorname{gmin}(t, U \cup V), U=O_{X}(t), V=$ $\operatorname{Min}\left(O_{G}(t)\right)$, then

$$
\Gamma^{\prime} \cup\{p\} \Rightarrow_{\Phi_{2}} \Gamma^{\prime} \cup\left\{t_{\mid u}^{\prime} \approx_{\mathrm{vf}} s_{\mid u}^{\prime} \mid u \in U\right\}
$$

Note that if both $s$ and $t$ are ground then common $\left(s^{\prime}, t^{\prime}\right)$ iff $s^{\prime}=t^{\prime}$ iff $s \downarrow t$. GG transformation of [14] is integrated with GT transformation in our new algorithm.

\section{Example 5.10:}

$$
\begin{aligned}
& \Gamma^{\prime} \cup\left\{\neg(\wedge(\mathrm{t}, \mathrm{t})) \approx_{\mathrm{vf}} \neg\left(\wedge\left(x^{\prime}, x^{\prime}\right)\right)\right\} \Rightarrow_{\Phi_{2}} \Gamma^{\prime} \cup\left\{x^{\prime} \approx_{\mathrm{vf}} \mathrm{t}\right\} \\
& \Gamma^{\prime} \cup\left\{\mathrm{t} \approx_{\mathrm{vf}} \mathrm{t}\right\} \Rightarrow_{\Phi_{2}} \Gamma^{\prime}
\end{aligned}
$$

\subsection{Final Stage}

Let $\Gamma$ be the output of Stage II. If $\Gamma$ is $\emptyset$-unifiable, then our algorithm answers ' $R$-unifiable', otherwise $\Gamma \Rightarrow_{\Phi}$ \{fail $\}$. (Note that our algorithm is a nondeterministic one.)

Since $\emptyset$-unifiability is equal to usual unifiability, any unification algorithm can be used [3], [7]. In fact, if $\Gamma$ satisfies (1) of the stop condition of Stage II then $\Gamma$ is in solved form, so that it is known that $\Gamma$ is unifiable iff $\Gamma$ is not cyclic [7]. The definition of cyclicity is given as follows (this definition is similar to that of [14]).
Definition 5.11: For $\Gamma$, a relation $\mapsto_{\Gamma}$ over $X$ is defined as follows: $x \mapsto_{\Gamma} y$ iff there exists $s \in S$ such that $x \approx_{\mathrm{vf}} s \in \Gamma$ and $y \in V(s)$ hold. Let $\mapsto_{\Gamma}^{+}$be the transitive closure of $\mapsto_{\Gamma}$. Then, $\Gamma$ is cyclic if there exists $x$ such that $x \mapsto_{\Gamma}^{+} x$.

We will prove later that $\Gamma$ is not cyclic if there exists a locally minimum $R$-unifier of $\Gamma$.

\section{Correctness condition of $\Phi$ :}

(1) $\Rightarrow_{\Phi_{1}}^{*} \cdot \Rightarrow_{\Phi_{2}}^{*}$ is terminating and finite branching, and

(2) $\Gamma_{0}=\left\{M_{0} \approx N_{0}\right\}$ is $R$-unifiable iff there exist $\Gamma_{1}$ and $\Gamma_{\mathrm{f}}$ such that $\Gamma_{0} \Rightarrow_{\Phi_{1}}^{*} \Gamma_{1} \Rightarrow_{\Phi_{2}}^{*} \Gamma_{\mathrm{f}}, \Gamma_{1}$ satisfies the stop condition of Stage I, $\Gamma_{\mathrm{f}}$ satisfies the one of Stage II, and $\Gamma_{\mathrm{f}}$ is $\emptyset$-unifiable (i.e., it is not cyclic and $\Gamma_{\mathrm{f}} \neq\{$ fail $\}$ ).

Note that since $\Phi$ is a nondeterministic algorithm, we need an exhaustive search of all the transformation sequences $\Rightarrow_{\Phi_{1}}^{*} \cdot \Rightarrow_{\Phi_{2}}^{*}$ from $\Gamma_{0}$, but it is ensured that we can decide whether $\Gamma_{0}$ is $R$-unifiable or not within finite time by (1) and (2) above.

Our algorithm can be easily transformed into one which produces a locally minimum $R$-unifier of $\Gamma_{0}$ iff $\Gamma_{0}$ is $R$-unifiable, since the information can be obtained when Substitution in Stage II is made.

\subsection{Example}

Let $\Gamma_{0}=\{\operatorname{nand}(\operatorname{nand}(x, \mathrm{t}), x) \approx x\}$. Our algorithm $\Phi$ can do the following transformations:

$$
\begin{aligned}
& \Gamma_{0} \Rightarrow_{V T}\left\{\text { nand }(x, \mathrm{t}) \triangleright \neg\left(\wedge\left(x^{\prime}, x^{\prime}\right)\right), x \approx x^{\prime}, \mathrm{t} \approx x\right\} \\
& \quad \text { by Example } 5.5(1) \\
& \Rightarrow_{T T}\left\{x \approx x^{\prime \prime}, \mathrm{t} \approx_{\mathrm{vf}} \mathrm{t}, x^{\prime \prime} \approx \mathrm{t},\right. \\
& \left.\neg\left(\wedge\left(x^{\prime \prime}, x^{\prime \prime}\right)\right) \triangleright \neg\left(\wedge\left(x^{\prime}, x^{\prime}\right)\right), x \approx x^{\prime}, \mathrm{t} \approx x\right\} \\
& \quad \text { by Example } 5.4(3) \\
& \Rightarrow_{T T}\left\{x \approx x^{\prime \prime}, \mathrm{t} \approx_{\mathrm{vf}} \mathrm{t}, x^{\prime \prime} \approx \mathrm{t}, \wedge\left(x^{\prime \prime}, x^{\prime \prime}\right) \triangleright \wedge\left(x^{\prime}, x^{\prime}\right),\right. \\
& \left.\quad x \approx x^{\prime}, \mathrm{t} \approx x\right\} \\
& \quad \text { by Example } 5.4(2) \\
& \Rightarrow_{T T}\left\{x \approx x^{\prime \prime}, \mathrm{t} \approx_{\mathrm{vf}} \mathrm{t}, x^{\prime \prime} \approx \mathrm{t}, x^{\prime \prime} \approx x^{\prime}, x \approx x^{\prime}, \mathrm{t} \approx x\right\} \\
& \quad \text { by Example } 5.4(2) \\
& \Rightarrow_{C o n v}\left\{x \approx_{\mathrm{vf}} x^{\prime \prime}, \mathrm{t} \approx_{\mathrm{vf}} \mathrm{t}, x^{\prime \prime} \approx_{\mathrm{vf}} \mathrm{t}, x^{\prime \prime} \approx_{\mathrm{vf}} x^{\prime}, x \approx_{\mathrm{vf}} x^{\prime},\right. \\
& \left.\quad x \approx_{\mathrm{vf}} \mathrm{t}\right\} \\
& \quad \text { by Example } 5.3 \\
& \Rightarrow_{S u b}\left\{\mathrm{t} \approx_{\mathrm{vf}} \mathrm{t}, x^{\prime \prime} \approx_{\mathrm{vf}} \mathrm{t}, x^{\prime \prime} \approx_{\mathrm{vf}} x^{\prime}\right\} \\
& \quad \text { by Example } 5.9 \\
& \Rightarrow_{S u b}\left\{\mathrm{t} \approx_{\mathrm{vf}} \mathrm{t}, \mathrm{t} \approx_{\mathrm{vf}} x^{\prime}\right\} \\
& \quad \text { by Example } 5.9 \\
& \Rightarrow_{S u b}\left\{\mathrm{t} \approx_{\mathrm{vf}} \mathrm{t}\right\} \\
& \Rightarrow_{G T} \emptyset \text { by Example } 5.10
\end{aligned}
$$

Obviously, $\emptyset$ satisfies the stop condition of Stage II and is $\emptyset$-unifiable. Hence, our algorithm decides that $\Gamma_{0}$ is $\mathrm{M}\left(R_{\mathrm{e}}\right)$ unifiable. By $\Rightarrow_{S u b}$ in this example, we obtain a substitution $\left\{x \rightarrow x^{\prime \prime}, x^{\prime \prime} \rightarrow \mathrm{t}, x^{\prime} \rightarrow \mathrm{t}\right\}$, that is, $\{x \rightarrow \mathrm{t}\}$ is an $\mathrm{M}\left(R_{\mathrm{e}}\right)-$ unifier. 
Note that $\Phi$ can also do the following transformations:

$$
\begin{aligned}
& \Gamma_{0} \Rightarrow_{V T}\{x \approx \operatorname{nand}(\mathrm{t}, x), \mathrm{t} \approx \operatorname{nand}(x, \mathrm{t})\} \\
& \quad \text { by Example } 5.5(2) \\
& \Rightarrow_{T T}\{x \approx \operatorname{nand}(\mathrm{t}, x), x \approx \mathrm{f}, \mathrm{f} \approx \mathrm{t}\} \\
& \quad \text { by Example } 5.4(1) \\
& \Rightarrow_{C o n v}\left\{x \approx_{\mathrm{vf}} \text { nand }(\mathrm{t}, x), x \approx_{\mathrm{vf}} \mathrm{f}, \mathrm{f} \approx_{\mathrm{vf}} \mathrm{t}\right\} \\
& \quad \text { by Example } 5.3 \\
& \Rightarrow_{S u b}\left\{\mathrm{f} \approx_{\mathrm{vf}} \text { nand }(\mathrm{t}, \mathrm{f}), \mathrm{f} \approx_{\mathrm{vf}} \mathrm{t}\right\} \\
& \Rightarrow\{\text { fail }\}
\end{aligned}
$$

Let us consider another example.

$$
\{\neg(x) \approx \wedge(x, x)\} \Rightarrow_{\Phi_{1}}\{\text { fail }\}
$$

Since no transformation is possible in Stage I, our algorithm produces $\{$ fail $\}$.

\section{Correctness of Algorithm $\Phi$}

In this section, we prove the lemmata needed to conclude the correctness of Algorithm $\Phi$ and the main theorem.

\subsection{Correctness of Stage I}

In order to prove the termination of Stage I, we define $\operatorname{size}(\Gamma)=\left(\#_{1}(\Gamma), \#_{2}(\Gamma)\right)$. Here

$$
\begin{aligned}
\#_{1}(\Gamma)= & \sqcup_{s \approx t \in \Gamma}\left(\mathrm{HD}_{G}(s) \sqcup \mathrm{HD}_{G}(t)\right) \\
& \sqcup\left(\sqcup_{s \triangleright t \in \Gamma} \mathrm{HD}_{G}(s)\right) \\
\#_{2}(\Gamma)= & \sqcup_{s \triangleright t \in \Gamma} \mathrm{HD}_{G}(t) .
\end{aligned}
$$

We use the lexicographic ordering $>_{\text {size }}$ to compare any $\Gamma, \Gamma^{\prime} \subseteq E_{0}$.

We explain the reason why we use the $\operatorname{size}(\Gamma)=$ $\left(\#_{1}(\Gamma), \#_{2}(\Gamma)\right)$. For each pair $p$ in $\Gamma$, if $p=s \approx t$ then $\mathrm{HD}_{G}(s) \sqcup \mathrm{HD}_{G}(t)$ is included in $\#_{1}(\Gamma)$, and if $p=s \triangleright t$ then $\mathrm{HD}_{G}(s)$ and $\mathrm{HD}_{G}(t)$ are included in $\#_{1}(\Gamma)$ and $\#_{2}(\Gamma)$, respectively. That is, we give the weight $\mathrm{HD}_{G}(t)$ a lower priority than the other weights. The reason is that when the TT transformation introduces new terms which are subterms of $\alpha$ for some rule $\alpha \rightarrow \beta$ in order to create new pairs added to $\Gamma$, the weight of these new terms are included in $\#_{2}(\Gamma)$, that is, they are given a lower priority, so that it becomes possible to avoid an increase of size $(\Gamma)$. Note that $s \approx_{\mathrm{vf}} t$ is counted neither for $\#_{1}$ nor for $\#_{2}$. Moreover, for the measures $\mathrm{HD}_{G}$ and $\#_{1}$, the following lemma holds.

\section{Lemma 6.1:}

(1) $s \gg_{\mathrm{HD}_{G}} s_{\mid v}$ for any $s \in S$ and $v \in O(s) \backslash\{\varepsilon\}$.

(2) $s \gg \mathrm{HD}_{G} s[t]_{v}$ for any $t \in G$ and any $v \in O(s)$ such that $s_{\mid v} \notin G$.

(3) $s \gg_{\mathrm{HD}_{G}} s \sigma$ for any $s$ and $\sigma=\{x \rightarrow t\}$ such that $x \in \mathrm{V}(s)$ and $t \notin S$.

(4) $s \gg_{\mathrm{HD}_{G}} s[\beta \rho]_{v}$ for any $\alpha \rightarrow \beta \in R$, substitution $\rho$ : $V(\beta) \rightarrow \operatorname{Psub}\left(s_{\mid v}\right) \cup \operatorname{Bud}\left(R_{\mathrm{C}}\right) \cup X$, and $v \in O_{D}(s)$ such as $s_{\mid v} \in S$.
(5) Let $p=s \approx t$ or $s \triangleright t$ where $s \in S$, then $\{p\} \gg_{\#_{1}}$ $\operatorname{Dec}(s, r)$ holds for any $r$ such as $\operatorname{root}(s)=\operatorname{root}(r)$.

\section{Proof}

(1) Since $\mathrm{HD}_{G}(s)=\mathrm{HD}_{G}\left(s_{\mid v}\right) \sqcup S_{\mathrm{m}}$ for some non-empty set $S_{\mathrm{m}}$, the proposition holds.

(2) By the definition of $\mathrm{HD}_{G}, \mathrm{HD}_{G}\left(s_{\mid v}\right) \neq \emptyset$ and $\mathrm{HD}_{G}(t)=$ $\emptyset$. By Lemma 2.6 (3), this proposition holds.

(3) If $s \in G$ then this proposition holds obviously. Otherwise, if $t \in G$ then $s \gg \mathrm{HD}_{G} s \sigma$ holds by (2). If $t \in X$ then $s=\mathrm{HD}_{G} s \sigma$ holds obviously.

(4) By Lemma 2.6 (3), it suffices to show that $s_{\mid v} \gg_{\mathrm{HD}_{G}} \beta \rho$. Since $\beta$ is a semi-constructor, $\mathrm{hD}_{G}(\beta \rho) \leq$ height $(\beta)+$ $\max \left\{\mathrm{hD}_{G}(r) \mid r \in \operatorname{Psub}\left(s_{\mid v}\right) \cup \operatorname{Bud}\left(R_{\mathrm{C}}\right) \cup X\right\}$. By $s_{\mid v} \in S$ and $v \in O_{D}(s), \mathrm{hD}_{G}\left(s_{\mid v}\right)>$ height $(\beta)+\mathrm{hD}_{G}(r)$ for any $r \in \operatorname{Psub}\left(s_{\mid v}\right) \cup \operatorname{Bud}\left(R_{\mathrm{C}}\right) \cup X$. Thus, this proposition holds.

(5) Let $k=\operatorname{ar}(\operatorname{root}(s))$. Note that $\#_{1}(\operatorname{Dec}(s, r)) \ll$

$\sqcup_{1 \leq i \leq k \wedge \eta_{i} \in S} \operatorname{HD}_{G}\left(s_{\mid i}\right) \sqcup \#_{1}\left(\left\{s_{\mid i} \approx r_{\mid i} \mid 1 \leq i \leq k, s_{\mid i} \notin\right.\right.$ $\left.\left.G, r_{\mid i} \notin S\right\}\right)$. By (1), $s \gg_{\mathrm{HD}_{G}} s_{\mid i}$ holds. By $s \in S$, if $r_{i i} \notin S$ then $s \gg \mathrm{HD}_{G} r_{i i}$ holds, since $\left|\mathrm{HD}_{G}(s)\right|>0$. Thus, this proposition holds.

We are ready to prove the termination of Stage I.

Lemma 6.2: $\quad$ Stage I is terminating and finite-branching.

Proof For every transformation $\Phi_{1}(\Gamma)=\left\{\Gamma_{1}, \cdots, \Gamma_{k}\right\}$ in Stage I, we prove that $\Gamma>_{\text {size }} \Gamma_{i}$ for every $i \in\{1, \cdots, k\}$ by showing the following table.

\begin{tabular}{l|ll} 
& $\#_{1}$ & $\#_{2}$ \\
\hline cases 1 and 2.b of TT & $\gg$ & \\
cases 2.a and 2.c of TT & $\gg$ & $\gg$ \\
case 1 of VT & $\gg$ & \\
case 2 of VT & $\gg$ & $\gg$
\end{tabular}

Let $\Gamma \Rightarrow_{\Phi_{1}} \tilde{\Gamma}$ and $\Gamma^{\prime}=\Gamma \backslash\{p\}$.

TT Transformation

1. Let $p=s \simeq t$ satisfy the TT condition.

a. Without loss of generality, we can assume that $s \in$ $S$. By Lemma 6.1(1), we have $\{p\} \gg_{\#_{1}}\left\{s_{\mid i} \approx\right.$ $\left.t_{\mid i} \mid 1 \leq i \leq \operatorname{ar}(\operatorname{root}(s))\right\}$, so that $\Gamma \gg_{\#_{1}} \tilde{\Gamma}$.

b. By Lemma 6.1(5), $\{p\} \gg_{\#_{1}} \operatorname{Dec}(s, \alpha)$ holds. Since $\beta$ is a semi-constructor, $\operatorname{hD}_{G}(\beta) \leq$ height $(\beta)$. By $s \in S$ and $\operatorname{root}(s) \in D, \mathrm{hD}_{G}(s)>$ height $(\beta)$. Thus, $\{p\} \gg_{\#_{1}}\{\beta \approx t\}$ holds.

c. We replace $\{p\}$ by $\left\{s^{\prime} \approx t\right\}$ for some term $s^{\prime} \in$ $\operatorname{Aux}(s)$, and $\left\{s^{\prime} \approx t\right\}$ is replaced by either $\left\{s_{\mid i}^{\prime} \approx\right.$ $\left.t_{\mid i} \mid 1 \leq i \leq \operatorname{ar}\left(\operatorname{root}\left(s^{\prime}\right)\right)\right\}$, where $\operatorname{root}\left(s^{\prime}\right)=\operatorname{root}(t)$, or $\{\beta \approx t\}$ for some right-ground rule $\alpha \rightarrow \beta$. And do a transformation on $t \approx \beta$ by case 1 .a or 1 .b of the TT transformation, i.e., $\{t \approx \beta\}$ is replaced by either $\left\{t_{\mid i} \approx \beta_{\mid i} \mid 1 \leq i \leq \operatorname{ar}(\operatorname{root}(t))\right\}$, where $\operatorname{root}(t)=\operatorname{root}(\beta)$, or $\operatorname{Dec}\left(t, \alpha^{\prime}\right) \cup\left\{\beta^{\prime} \approx t\right\}$ for some rule $\alpha^{\prime} \rightarrow \beta^{\prime}$. In either case, the $\#_{1}$-value strictly 
decreases by the arguments of case 1.a and 1.b since $t \in S$. Note that $\{p\}=$ size $\left\{s^{\prime} \approx t\right\}==_{\text {size }}$ $\{\beta \approx t\}$ since $s^{\prime}, \beta \in G$.

2. Let $p=s \triangleright t$ satisfy the TT condition.

a. If $s \in S$, then the $\#_{1}$-value strictly decreases by Lemma 6.1(5). Otherwise, $s \in G$ holds and $\operatorname{Dec}(s, t)=\left\{s_{\mid i} \triangleright t_{\mid i} \mid 1 \leq i \leq \operatorname{ar}(\operatorname{root}(s)), t_{\mid i} \in S\right\} \cup$ $\left\{t_{\mid i} \approx_{\mathrm{vf}} s_{\mid i} \mid 1 \leq i \leq \operatorname{ar}(\operatorname{root}(s)), t_{\mid i} \notin S, s_{\mid i} \in G\right\}$, so that $\{p\}=_{\#_{1}} \operatorname{Dec}(s, t)$ since $s \in G$. By $t \in S$, $\{p\} \gg_{\#_{2}} \operatorname{Dec}(s, t)$ holds by the definition of $\#_{2}$ and Lemma 6.1 (1).

b. $\{p\} \Rightarrow_{\Phi_{1}} \operatorname{Dec}\left(s^{\prime}, \alpha^{\prime} \rho\right) \cup\left\{x \rho \approx x^{\prime} \rho \mid x \in \mathrm{V}(\alpha), x \equiv\right.$ $\left.x^{\prime}\right\} \cup\left\{s[\beta \rho]_{v} \triangleright t\right\}$. Here, $v \in O(s)$ such that $s_{\mid v} \in S$, $s^{\prime}=s_{\mid v}\left[s_{1} \cdots, s_{n}\right]_{\left(u_{1}, \cdots, u_{n}\right)}$ where $\operatorname{Min}\left(O_{G}\left(s_{\mid v}\right)\right)=$ $\left\{u_{1}, \cdots, u_{n}\right\}$ and $s_{i} \in \operatorname{Aux}\left(s_{\mid v u_{i}}\right)$ for $i \in\{1, \cdots, n\}$, $\alpha^{\prime}=\operatorname{linearize}(\alpha)$ where $\alpha \rightarrow \beta \in R$, and $\rho \in \overline{\operatorname{BudMap}}_{R}\left(s^{\prime}, \alpha\right)$. Note that $s \gg_{\mathrm{HD}_{G}} s^{\prime}$ holds. By Lemma 6.1(5), $\{p\} \gg_{\#_{1}} \operatorname{Dec}\left(s^{\prime}, \alpha^{\prime} \rho\right)$. By Lemma 6.1 (4), $\{p\} \gg_{\#_{1}}\left\{x \rho \approx x^{\prime} \rho \mid x \in \mathrm{V}(\alpha), x \equiv\right.$ $\left.x^{\prime}\right\} \cup\left\{s[\beta \rho]_{v} \triangleright t\right\}$.

c. We replace $\{p\}$ by $\left\{s^{\prime} \triangleright t\right\}$ for some term $s^{\prime} \in$ $\operatorname{Aux}(s)$, and $\left\{s^{\prime} \triangleright t\right\}$ is replaced by either $\operatorname{Dec}\left(s^{\prime}, t\right)$, where $\operatorname{root}(t)=\operatorname{root}(\beta)$, or $\{\beta \triangleright t\}$ for some rightground rule $\alpha \rightarrow \beta$. And transform $\beta \triangleright t$ by case 2.a of the TT transformation, i.e., if $\operatorname{root}\left(s^{\prime}\right)=\operatorname{root}(t)$ then $\{\beta \triangleright t\}$ is replaced by $\operatorname{Dec}(\beta, t)$. In either case, the size strictly decreases by the same arguments as those of case 2.a. Note that $\{p\}={ }_{\text {size }}$ $\left\{s^{\prime} \triangleright t\right\}==_{\text {size }}\{\beta \triangleright t\}$.

\section{VT Transformation}

1. Let $p=x \simeq s$ with $s \in S$.

a. $\{p\} \Rightarrow_{\Phi_{1}} \operatorname{Dec}\left(s_{\mid v}, \alpha\right) \cup\left\{s[\beta]_{v} \approx x\right\}$ where $\alpha \rightarrow \beta \in$ $R, v \in O(s)$ and $s_{\mid v} \in S$. By Lemma 6.1 (4), (5), we have $\{s \approx x\} \gg_{\#_{1}} \operatorname{Dec}\left(s_{\mid v}, \alpha\right) \cup\left\{s[\beta]_{v} \approx x\right\}$. Thus, the $\#_{1}$-value strictly decreases.

b. If $s[c]_{v} \notin G$ then $\{p\} \Rightarrow_{\Phi_{1}}\left\{x \approx s[c]_{v}, c \approx s_{\mid v}\right\}$ where $c \in F_{0}, v \in O(s)$ and $s_{\mid v} \in S$. By Lemma 6.1 (2), we have $\{x \approx s\} \gg_{\#_{1}}\{x \approx$ $\left.s[c]_{v}\right\}$. By Lemma 6.1(1) and $x \gg_{\mathrm{hD}_{G}} c$, we

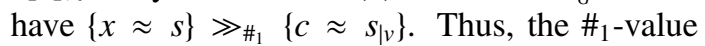
strictly decreases. If $s[c]_{v} \in G$, then $\{p\} \Rightarrow_{\Phi_{1}}$ $\left\{x \approx_{\text {vf }} s[c]_{v}, c \approx s_{\mid v}\right\}$ where $v \in O(s)$ and $s_{\mid v} \in S$. Since $\#_{1}\left(\left\{x \approx_{\mathrm{vf}} s[c]_{v}, c \approx s_{\mid v}\right\}\right)=\operatorname{HD}_{G}\left(s_{\mid v}\right)$, the $\#_{1^{-}}$ value strictly decreases.

2. Let $p=x \triangleright s$ and $s_{\mid v} \in S$. If $s[c]_{v} \notin G$ then $\{p\} \Rightarrow_{\Phi_{1}}$ $\left\{x \triangleright s[c]_{v}, c \triangleright s_{\mid v}\right\}$. Then, $\{p\}=\#_{1}\left\{x \triangleright s[c]_{v}, c \triangleright s_{\mid v}\right\}$ by the definition of $\#_{1}$. By Lemma 6.1 (2), we have $\{p\} \gg_{\#_{2}}\left\{x \triangleright s[c]_{v}\right\}$ By Lemma 6.1 (1) and $x \gg_{\mathrm{hD}_{G}} c$, we have $\{p\} \gg_{\#_{2}}\left\{c \triangleright s_{\mid v}\right\}$. Thus, the $\#_{1}$-value is unchanged, but the $\#_{2}$-value strictly decreases. If $s[c]_{v} \in G$, then $\{p\} \Rightarrow_{\Phi_{1}}\left\{x \approx_{\mathrm{vf}} s[c]_{v}, c \triangleright s_{\mid v}\right\}$ where $v \in O(s)$. Since $\#_{1}(\{p\})=\{0\}_{\mathrm{m}}$ and $\#_{1}\left(\left\{x \approx_{\mathrm{vf}} s[c]_{v}, c \triangleright s_{\mid v}\right\}\right)=\emptyset$, the $\#_{1}$-value strictly decreases.
Moreover, if $\Gamma$ is a finite set, then $k$ is finite, i.e., Stage $\mathrm{I}$ is finite-branching. Thus, this lemma holds.

\section{Lemma 6.3:}

(1) Stage I is valid.

(2) If $\Gamma \subseteq E_{0}$ is $R$-unifiable and does not satisfy the stop condition of Stage I, then $\Phi_{1}(\Gamma) \neq \emptyset$.

Proof To show that $\Phi_{1}$ satisfies the validity condition (V1) and Lemma 6.3 (2), let $\theta$ be a locally minimum $R$-unifier of $\Gamma$. We first show that if $p=s \simeq t$ or $p=s \triangleright t$ in $\Gamma$ satisfies the TT condition, then $\Phi_{1}$ can do a TT transformation $\Gamma \Rightarrow_{\Phi_{1}} \tilde{\Gamma}$ such that there exists a locally minimum $R$-unifier $\theta^{\prime}$ of $\tilde{\Gamma}$ consistent with $\theta$. Next, we show that in the remaining case, i.e., if there exists no $p$ in $\Gamma$ satisfying the TT condition, $\Phi_{1}$ can do a VT transformation or Conversion $\Gamma \Rightarrow{ }_{\Phi_{1}} \tilde{\Gamma}$ such that there exists a locally minimum $R$-unifier $\theta^{\prime}$ of $\tilde{\Gamma}$ consistent with $\theta$. It follows that $\Phi_{1}$ satisfies (V1) and Lemma $6.3(2)$. It remains that $\Phi_{1}$ satisfies (V2). The proof is straightforward as explained below. Now we prove this lemma. We assume that $p \in \Gamma$.

\section{TT Transformation}

Let $p=s \simeq t$ satisfy the TT condition, i.e., $s, t \notin X$ and either $s \notin G$ or $t \notin G$. Let $k=\operatorname{ar}(\operatorname{root}(s))$. Then, since $\theta$ is a locally minimum unifier of $p$ and $R$ is confluent, we have a sequence $\gamma: s \theta \downarrow t \theta$. There are two cases: (1) $\gamma$ is $\varepsilon$-invariant and (2) $\varepsilon \in \mathcal{R}(\gamma)$.

In case (1), we have $\operatorname{root}(s)=\operatorname{root}(t)$ and for any $i \in$ $\{1, \cdots, k\}, s_{\mid i} \theta \downarrow t_{\mid i} \theta$. Thus, $\Phi_{1}$ can do a transformation by case 1.a of the TT transformation:

$$
\Gamma\left(=\Gamma^{\prime} \cup\{p\}\right) \Rightarrow_{\Phi_{1}} \Gamma^{\prime} \cup\left\{s_{\mid i} \approx t_{\mid i} \mid 1 \leq i \leq k\right\}(=\tilde{\Gamma})
$$

Hence, $\tilde{\Gamma}$ satisfies the required condition: locally minimum $\theta$ is also an $R$-unifier of $\tilde{\Gamma}$. Thus, the validity condition (V1) holds.

Conversely, if $\theta^{\prime}$ is an $R$-unifier of $\operatorname{Core}(\tilde{\Gamma})$, then there exist sequences $\gamma_{i}: s_{\mid i} \theta^{\prime} \leftrightarrow^{*} t_{\mid i} \theta^{\prime}$ for any $i \in\{1, \cdots, k\}$. Since $\operatorname{root}(s)=\operatorname{root}(t)$, there exists a sequence $s \theta^{\prime} \leftrightarrow^{*} t \theta^{\prime}$, i.e., $\theta^{\prime}$ is an $R$-unifier of Core $(\{p\})$. So, (V2) holds.

In case (2), we first consider the case of $s \notin G$. In this case, without loss of generality, we assume that

$$
\gamma: s \theta \rightarrow^{*} \alpha \sigma \rightarrow \beta \sigma \downarrow t \theta
$$

for some rule $\alpha \rightarrow \beta$ and substitution $\sigma$. (For the other case, exchange $s$ and $t$.) Let the above $\varepsilon$-reduction $\alpha \sigma \rightarrow \beta \sigma$ be leftmost, i.e., the subsequence $\gamma^{\prime}$ (of $\gamma$ ): $s \theta \rightarrow^{*} \alpha \sigma$ is $\varepsilon$-invariant. Hence, $\operatorname{root}(s)=\operatorname{root}(\alpha)$ and for any $i \in$ $\{1, \cdots, k\}, s_{\mid i} \theta \rightarrow^{*} \alpha_{\mid i} \sigma$ holds. Thus, $\Phi_{1}$ can do a transformation by case $1 . b$ :

$$
\Gamma\left(=\Gamma^{\prime} \cup\{p\}\right) \Rightarrow_{\Phi_{1}} \Gamma^{\prime} \cup \operatorname{Dec}(s, \alpha) \cup\{\beta \approx t\}(=\tilde{\Gamma})
$$

If $\sigma$ is not locally minimum, then let $\sigma^{\prime}$ be locally minimum such that $x \sigma^{\prime} \leftrightarrow^{*} x \sigma$ for every $x \in \operatorname{Dom}(\sigma)$. The existence of $\sigma^{\prime}$ is obvious by the definition of local minimum 
property: let $x \sigma^{\prime}$ be the minimum term in $\mathcal{L}(x \sigma)$. Here, we assume that $\operatorname{Dom}(\theta) \cap \operatorname{Dom}\left(\sigma^{\prime}\right)=\emptyset$. So, let $\theta^{\prime}=\theta \cup \sigma^{\prime}$, i.e., $\operatorname{Dom}\left(\theta^{\prime}\right)=\operatorname{Dom}(\theta) \cup \operatorname{Dom}\left(\sigma^{\prime}\right)$ and $x \theta^{\prime}=x \theta$ for every $x \in \operatorname{Dom}(\theta)$ and $y \theta^{\prime}=y \sigma^{\prime}$ for every $y \in \operatorname{Dom}\left(\sigma^{\prime}\right)$. Note that $s \theta^{\prime} \stackrel{>\varepsilon}{\rightarrow}{ }^{*} \alpha \sigma \stackrel{\geq O_{X}(\alpha)}{\leftrightarrow}{ }^{*} \alpha \theta^{\prime} \rightarrow \beta \theta^{\prime} \downarrow t \theta^{\prime}$. It is obvious that $\theta^{\prime}$ is locally minimum and $\theta^{\prime}$ is an $R$-unifier of $\tilde{\Gamma}$ by the definition of $\operatorname{Dec}(s, \alpha)$. Hence, the validity condition (V1) holds.

Conversely, if $\theta^{\prime}$ is an $R$-unifier of $\operatorname{Core}(\tilde{\Gamma})$, then there exist sequences $\gamma_{i}: s_{\mid i} \theta^{\prime} \leftrightarrow^{*} \alpha_{\mid i} \theta^{\prime}$ for any $i \in$ $\{1, \cdots, \operatorname{ar}(\operatorname{root}(s))\}$ and $\gamma^{\prime}: \beta \theta^{\prime} \leftrightarrow^{*} t \theta^{\prime}$. Since $\operatorname{root}(s)=$ $\operatorname{root}(\alpha)$, there exists a sequence $s \theta^{\prime} \leftrightarrow^{*} \alpha \theta^{\prime}$. So there exists a sequence $s \theta^{\prime} \leftrightarrow^{*} t \theta^{\prime}$, i.e., $\theta^{\prime}$ is an $R$-unifier of Core $(\{p\})$. So, (V2) holds.

The remaining case is that $s \in G$. In this case, $t \in S$. There exists $s^{\prime} \in \operatorname{Aux}(s)$ such that $\gamma^{\prime}: s^{\prime} \rightarrow_{R_{\mathrm{rg}}}^{*} \leftarrow^{*} t \theta$, by Lemma 3.10 (2). If $\gamma^{\prime}$ is $\varepsilon$-invariant, we can do a transformation by case 1.c.i. The proof is similar to that of case 1.a since $s \leftrightarrow^{*} s^{\prime}$ by Lemma 3.10(1). Otherwise, we have

$$
\gamma^{\prime}: s^{\prime} \rightarrow_{R_{\mathrm{rg}}}^{*} \alpha \sigma \rightarrow \beta \downarrow t \theta
$$

for some right-ground rule $\alpha \rightarrow \beta$ and substitution $\sigma$. Let the above $\varepsilon$-reduction $\alpha \sigma \rightarrow \beta$ be rightmost, i.e., in the subsequence $\gamma^{\prime \prime}$ (of $\gamma$ ): $\beta \downarrow t \theta$ there is no $\varepsilon$-reduction from left to right. Note that $s^{\prime} \rightarrow_{R_{\mathrm{rg}}}^{+} \beta$. Thus, $\Phi_{1}$ can do a transformation by case 1.c.ii:

$$
\Gamma\left(=\Gamma^{\prime} \cup\{p\}\right) \Rightarrow_{\Phi_{1}} \Gamma^{\prime} \cup\{\beta \approx t\}(=\tilde{\Gamma})
$$

Obviously, $\theta$ is a locally minimum $R$-unifier of $\tilde{\Gamma}$. It follows that $\Phi_{1}$ can transform $t \approx \beta$ by case 1 .a or 1 .b of the TT transformation (i.e., $\Phi_{1}$ can do a transformation by case 1 .a if $\gamma^{\prime \prime}: t \theta \downarrow \beta$ is $\varepsilon$-invariant, otherwise case 1.b). In either case, (V1) holds.

Conversely, if $\theta^{\prime}$ is an $R$-unifier of $\operatorname{Core}(\tilde{\Gamma})$, then there exists a sequence $\gamma^{\prime}: \beta \leftrightarrow^{*} t \theta^{\prime}$. Since $s \downarrow s^{\prime}$ and $s^{\prime} \rightarrow^{+} \beta$, there exists a sequence $s \leftrightarrow^{*} t \theta^{\prime}$, i.e., $\theta^{\prime}$ is an $R$-unifier of Core $(\{p\})$. So, (V2) holds.

Let $p=s \triangleright t$ satisfy the TT condition, i.e., $s \notin X$ and $t \in S$. Let $k=\operatorname{ar}(\operatorname{root}(s))$. Since $\theta$ is a locally minimum $R$-unifier of $p$, there exists a sequence $\gamma: s \theta \rightarrow^{*} r \stackrel{\geq O_{X}(t)}{\leftrightarrow}{ }^{*} t \theta$ for some term $r$. There are two cases: (1) $\gamma$ is $\varepsilon$-invariant and (2) $\varepsilon \in \mathcal{R}(\gamma)$.

In case (1), we have $\operatorname{root}(s)=\operatorname{root}(t)$ and for any $\geq O_{X}\left(t_{i}\right)$

$i \in\{1, \cdots, k\}, s_{\mid i} \theta \rightarrow^{*} r_{\mid i} \stackrel{\Theta^{*}}{\leftrightarrow} t_{\mid i} \theta$. Thus, $\Phi_{1}$ can do a transformation by case 2 .a of the TT transformation:

$$
\Gamma\left(=\Gamma^{\prime} \cup\{p\}\right) \Rightarrow_{\Phi_{1}} \Gamma^{\prime} \cup \operatorname{Dec}(s, t)(=\tilde{\Gamma})
$$

It is obvious that $\theta$ is also a locally minimum $R$-unifier of $\tilde{\Gamma}$. Thus, (V1) holds.

Conversely, if $\theta^{\prime}$ is an $R$-unifier of Core $(\tilde{\Gamma})$, then there exist sequences $\gamma_{i}: s_{\mid i} \theta^{\prime} \leftrightarrow^{*} t_{\mid i} \theta^{\prime}$ for any $i \in\{1, \cdots, k\}$. Since $\operatorname{root}(s)=\operatorname{root}(t)$, there exists a sequence $s \theta^{\prime} \leftrightarrow^{*} t \theta^{\prime}$, i.e., $\theta^{\prime}$ is an $R$-unifier of Core $(\{p\})$. So, (V2) holds.

In case (2), we first consider the case of $s \notin G$. we can assume that

$$
\gamma: s \theta \rightarrow^{*} s \theta[\alpha \sigma]_{v} \rightarrow s \theta[\beta \sigma]_{v} \rightarrow^{*} r \stackrel{\geq O_{X}(t)}{\leftrightarrow}{ }^{*} t \theta
$$

for some rule $\alpha \rightarrow \beta$, substitution $\sigma$, and some $v \in O(s)$ such as $s_{\mid v} \in S$. Let the above $v$-reduction $s \theta[\alpha \sigma]_{v} \rightarrow$ $s \theta[\beta \sigma]_{v}$ be first reduction at non-ground and non-variable position of $s$, i.e., $s \theta \rightarrow^{*} s \theta[\alpha \sigma]_{v}$ is $O_{X}(s)$-frontier. Let $\operatorname{Min}\left(O_{G}\left(s_{\mid v}\right)\right)=\left\{u_{1}, \cdots, u_{n}\right\}$. By Lemma 3.10(2), for every $i \in\{1, \cdots, n\}$, if $s_{\mid v u_{i}} \rightarrow^{*} \alpha \sigma_{\mid u_{i}}$ then there exists $s_{i} \in \operatorname{Aux}\left(s_{\mid v u_{i}}\right)$ such that $s_{i} \rightarrow_{R_{\mathrm{rg}}}^{*} \alpha \sigma_{\mid u_{i}}$. So, let $s^{\prime}=$ $s_{\mid v}\left[s_{1}, \cdots, s_{n}\right]_{\left(u_{1}, \cdots, u_{n}\right)}$. By Lemma $4.12(2)$, there exist $\rho \in$ $\overline{\operatorname{BudMap}}_{R}\left(s^{\prime}, \alpha\right)$ and a locally minimum substitution $\theta^{\prime}$ such that $s^{\prime} \theta \stackrel{\geq O_{X}\left(s^{\prime}\right) \cup \operatorname{Min}\left(O_{G}\left(s^{\prime}\right)\right)}{\rightarrow} \alpha^{*} \rho \theta^{\prime} \stackrel{\geq O_{X}\left(\alpha^{\prime}\right)}{\rightarrow} \alpha \sigma$ and $\beta \rho \theta^{\prime} \stackrel{\geq O_{X}(\beta)}{\rightarrow}$ $\beta \sigma$, where $\alpha^{\prime}=$ linearize $(\alpha)$. Since $\alpha^{\prime} \rho \theta^{\prime} \stackrel{\geq O_{X}\left(\alpha^{\prime}\right)}{\rightarrow} \alpha \sigma$, $x \rho \theta^{\prime} \rightarrow^{*} x \sigma \leftarrow^{*} x^{\prime} \rho \theta^{\prime}$ holds for every $x \in \mathrm{V}(\alpha)$ and $x \equiv x^{\prime}$. Since $s^{\prime} \theta \rightarrow^{*} \alpha^{\prime} \rho \theta^{\prime}$ is $O_{X}\left(s^{\prime}\right)$-frontier and $s^{\prime} \in S$, $\operatorname{root}\left(s^{\prime}\right)=\operatorname{root}\left(\alpha^{\prime} \rho\right)$ and for any $i \in\left\{1, \cdots, \operatorname{ar}\left(\operatorname{root}\left(s^{\prime}\right)\right\}\right.$, $s_{\mid i}^{\prime} \theta \rightarrow{ }^{*} \alpha^{\prime} \rho_{\mid i} \theta^{\prime}$ holds. Thus, $\Phi_{1}$ can do a transformation by case 2.b:

$$
\begin{aligned}
& \Gamma\left(=\Gamma^{\prime} \cup\{p\}\right) \\
& \Rightarrow_{\Phi_{1}} \Gamma^{\prime} \cup \operatorname{Dec}\left(s^{\prime}, \alpha^{\prime} \rho\right) \\
& \cup\left\{x \rho \approx x^{\prime} \rho \mid x \in \mathrm{V}(\alpha), x \equiv x^{\prime}\right\} \\
& \cup\left\{s[\beta \rho]_{v} \triangleright t\right\}(=\tilde{\Gamma})
\end{aligned}
$$

$\theta^{\prime}$ is a locally minimum $R$-unifier of $\tilde{\Gamma}$. Thus, (V1) holds.

Conversely, if $\theta^{\prime}$ is an $R$-unifier of $\operatorname{Core}(\tilde{\Gamma})$, then there exist sequences $\gamma_{i}: s_{\mid i}^{\prime} \theta^{\prime} \leftrightarrow^{*} \alpha^{\prime} \rho_{\mid i} \theta^{\prime}$ for any $i \in$ $\left\{1, \cdots, \operatorname{ar}\left(\operatorname{root}\left(s^{\prime}\right)\right)\right\}, \gamma^{\prime}: s[\beta \rho]_{v} \theta^{\prime} \leftrightarrow^{*} t \theta^{\prime}$, and $\gamma_{x^{\prime}}: x \rho \theta^{\prime} \leftrightarrow^{*}$ $x^{\prime} \rho \theta^{\prime}$ for any $x \in \mathrm{V}(\alpha)$ and $x \equiv x^{\prime}$. Since $\operatorname{root}\left(s^{\prime}\right)=\operatorname{root}(\alpha)$, there exists a sequence $s\left[s^{\prime}\right]_{v} \theta^{\prime} \leftrightarrow^{*} s^{\prime}\left[\alpha^{\prime} \rho\right]_{v} \theta^{\prime}$. Since $R$ is confluent and $x \rho \theta^{\prime} \leftrightarrow^{*} x^{\prime} \rho \theta^{\prime}$ holds for any $x \in \mathrm{V}(\alpha)$ and $x \equiv x^{\prime}$, there exists a substitution $\sigma^{\prime}: \mathrm{V}(\alpha) \rightarrow T$ such that $s\left[\alpha^{\prime} \rho\right]_{v} \theta^{\prime} \leftrightarrow^{*} s \theta^{\prime}\left[\alpha \sigma^{\prime}\right]_{v} \rightarrow s \theta^{\prime}\left[\beta \sigma^{\prime}\right]_{v}$ and $s \theta^{\prime}[\beta \rho]_{v} \leftrightarrow^{*}$ $s \theta^{\prime}\left[\beta \sigma^{\prime}\right]_{v}$. Since $s \theta^{\prime} \leftrightarrow^{*} s\left[s^{\prime}\right]_{v} \theta^{\prime}$, there exists a sequence $s \theta^{\prime} \leftrightarrow^{*} t \theta^{\prime}$, i.e., $\theta^{\prime}$ is an $R$-unifier of Core $(\{p\})$. So, (V2) holds.

The remaining case is that $s \in G$. There exists $s^{\prime} \in$ Aux $(s)$ such that $\gamma^{\prime}: s^{\prime} \rightarrow_{R_{\mathrm{rg}}}^{*} r \stackrel{\geq O_{X}(t)}{\leftrightarrow}{ }^{*} t \theta$, by Lemma $3.10(2)$. If $\gamma^{\prime}$ is $\varepsilon$-invariant, we can do a transformation by case 2.c.i. The proof is similar to that of case 1.a since $s \leftrightarrow^{*} s^{\prime}$ by Lemma 3.10 (1). Otherwise, we assume that

$$
\gamma^{\prime}: s^{\prime} \rightarrow_{R_{\mathrm{rg}}}^{*} \alpha \sigma \rightarrow \beta \rightarrow^{*} r \stackrel{\geq O_{X}(t)}{\leftrightarrow}{ }^{*} t \theta
$$

for some right-ground rule $\alpha \rightarrow \beta$ and substitution $\sigma$. In this case, let the $\varepsilon$-reduction $\alpha \sigma \rightarrow \beta$ in the above sequence $\gamma$ be rightmost, i.e., in the subsequence $\gamma^{\prime \prime}$ (of $\gamma$ ): $\beta \rightarrow^{*} r \stackrel{\geq O_{X}(t)}{\leftrightarrow}$ $t \theta$ there is no $\varepsilon$-reduction. Since $s^{\prime} \rightarrow_{R_{\mathrm{rg}}}^{+} \beta$ holds by $\gamma, \Phi_{1}$ can do a transformation by case 2.c:

$$
\Gamma\left(=\Gamma^{\prime} \cup\{p\}\right) \Rightarrow_{\Phi_{1}} \Gamma^{\prime} \cup\{\beta \triangleright t\}(=\tilde{\Gamma})
$$

Obviously, $\theta$ is a locally minimum $R$-unifier of $\tilde{\Gamma}$. Moreover $\Phi_{1}$ can transform $\beta \triangleright t$ by case 2 .a of the TT transformation, 
since $\gamma^{\prime \prime}: \beta \rightarrow^{*} r \stackrel{\geq O_{X}(t)}{\leftrightarrow}{ }^{*} t \theta$ is $\varepsilon$-invariant. Thus, (V1) holds.

Conversely, if $\theta^{\prime}$ is an $R$-unifier of $\operatorname{Core}(\tilde{\Gamma})$, then there exists a sequence $\gamma^{\prime}: \beta \leftrightarrow^{*} t \theta^{\prime}$. Since $s \downarrow s^{\prime}$ and $s^{\prime} \rightarrow^{+} \beta$, there exists a sequence $s \leftrightarrow^{*} t \theta^{\prime}$, i.e., $\theta^{\prime}$ is an $R$-unifier of Core $(\{p\})$. So, (V2) holds.

By the above arguments, if $\theta$ is a locally minimum $R$ unifier of $\Gamma$ and there exists $p \in \Gamma$ satisfying the TT condition, then we can perform a TT transformation $\Gamma \Rightarrow_{\Phi_{1}} \tilde{\Gamma}$ such that there exists a locally minimum $R$-unifier $\theta^{\prime}$ of $\tilde{\Gamma}$ consistent with $\theta$.

Thus, this lemma holds in this case.

\section{VT Transformation and Conversion}

Let every $s \approx t, s \triangleright t \in \Gamma$ do not satisfy the TT condition, i.e., every $s \approx t, s \triangleright t \in \Gamma \backslash(G \cup X) \times(G \cup X)$ satisfies the VT condition. Since $\theta$ is a locally minimum $R$-unifier of $\Gamma$, for every $x \simeq s$ and $x \triangleright s$ in $\Gamma, \gamma: x \theta \leftrightarrow^{*} s \theta$ holds. For every such $\gamma$, if $v \mid u$ or $u \leq v$ for every $v \in \mathcal{R}(\gamma)$ and $u \in O_{X}(s)$ (i.e., $O_{X}(s)$ is a frontier in $\gamma$, so that $x \approx_{\text {vf }} s$ is $R$-unifiable), then $\Phi_{1}$ can do a Conversion

$$
\Gamma \Rightarrow_{\Phi_{1}} \operatorname{Conv}(\Gamma)
$$

and $\theta$ is a locally minimum $R$-unifier of $\operatorname{Conv}(\Gamma)$. Thus, (V1) holds. Conversely, since $\operatorname{Core}(\Gamma)=\operatorname{Core}(\operatorname{Conv}(\Gamma))$, (V2) holds.

Otherwise, i.e., there exists $p=x \simeq s$ (or $p=x \triangleright s)$ in $\Gamma$ such that $\gamma: x \theta \leftrightarrow^{*} s \theta$ (or $\gamma: x \theta \rightarrow^{*} r \stackrel{\geq O_{X}(s)}{\leftrightarrow}$ * $s \theta$ for some $r$ ) and $v<u$ for some $v \in \operatorname{Min}(\mathcal{R}(\gamma))$ and $u \in O_{X}(s)$. So, $s_{\mid v} \in S$. We first consider the case of $p=x \simeq s$. Then, there exist sequences $\gamma^{\prime}: s \theta \rightarrow^{*} t$ and $\gamma^{\prime \prime}: x \theta \rightarrow^{*} t$ for some $t$. There are two cases (a) $v \in \mathcal{R}\left(\gamma^{\prime}\right)$ and (b) $v \in \mathcal{R}\left(\gamma^{\prime \prime}\right) \backslash \mathcal{R}\left(\gamma^{\prime}\right)$. If $v \in \mathcal{R}\left(\gamma^{\prime}\right)$, we must have $\gamma: s \theta \rightarrow^{*} s \theta[\alpha \sigma]_{v} \rightarrow s \theta[\beta \sigma]_{v} \downarrow x \theta$ for some rule $\alpha \rightarrow \beta$ and some substitution $\sigma$. Let the above $v$-reduction $s \theta[\alpha \sigma]_{v} \rightarrow s \theta[\beta \sigma]_{v}$ be leftmost, i.e., the subsequence $\delta$ (of $\gamma): s \theta \rightarrow{ }^{*} s \theta[\alpha \sigma]_{v}$ is $v$-invariant. Hence, $\operatorname{root}\left(s_{\mid v}\right)=\operatorname{root}(\alpha)$ and for any $i \in\left\{1, \cdots, \operatorname{ar}\left(\operatorname{root}\left(s_{\mid v}\right)\right)\right\}, s_{\mid v i} \theta \rightarrow^{*} \alpha_{\mid i} \sigma$ holds. Thus, $\Phi_{1}$ can do a transformation

$$
\Gamma\left(=\Gamma^{\prime} \cup\{p\}\right) \Rightarrow_{\Phi_{1}} \Gamma^{\prime} \cup \operatorname{Dec}\left(s_{\mid v}, \alpha\right) \cup\left\{s[\beta]_{v} \approx x\right\}(=\tilde{\Gamma})
$$

If $\sigma$ is not locally minimum, then let $\sigma^{\prime}$ be a locally minimum $R$-unifier such that for any $y \in \operatorname{Dom}(\sigma), y \sigma^{\prime} \leftrightarrow^{*} y \sigma$ holds as in the proof concerning the TT transformation. Let $\theta^{\prime}=\theta \cup \sigma^{\prime}$. Then $\theta^{\prime}$ is a locally minimum $R$-unifier of $\geq O_{X}\left(\alpha_{\mid i}\right)$

$\operatorname{Dec}\left(s_{\mid v}, \alpha\right)$, since $s_{\mid v i} \theta^{\prime} \rightarrow^{*} \alpha_{\mid i} \sigma \stackrel{O_{X}\left(\alpha_{\mid i}\right)}{\leftrightarrow} \alpha_{\mid i} \sigma^{\prime}$. Substitution $\theta^{\prime}$ is also a locally minimum $R$-unifier of $s[\beta]_{v} \approx x$, since $s[\beta]_{v} \theta^{\prime} \downarrow x \theta^{\prime}$. Hence, $\theta^{\prime}$ is a locally minimum $R$-unifier of $\tilde{\Gamma}$. Thus, (V1) holds. Conversely, if $\theta^{\prime}$ is an $R$-unifier of Core $(\tilde{\Gamma})$, then there exist sequences $\gamma_{i}: s_{\mid v i} \theta^{\prime} \leftrightarrow^{*} \alpha_{\mid i} \theta^{\prime}$ for any $i \in\{1, \cdots, \operatorname{ar}(\operatorname{root}(s))\}$ and $\gamma^{\prime}: s[\beta]_{v} \theta^{\prime} \leftrightarrow^{*} x \theta^{\prime}$. Since $\operatorname{root}\left(s_{\mid v}\right)=\operatorname{root}(\alpha)$, there exists a sequence $s \theta^{\prime} \leftrightarrow \leftrightarrow^{*} s[\alpha]_{v} \theta^{\prime}$, so that there exists a sequence $s \theta^{\prime} \leftrightarrow^{*} x \theta^{\prime}$, i.e., $\theta^{\prime}$ is an $R$ unifier of Core $(\{p\})$. So, (V2) holds.
The remaining case is that $v \notin \mathcal{R}\left(\gamma^{\prime}\right)$, i.e., $v \in \mathcal{R}\left(\gamma^{\prime \prime}\right)$. By $v \in \operatorname{Min}(\mathcal{R}(\gamma))$, we must have $s \theta_{\mid v} \rightarrow^{*} t_{\mid v}$. By minimum of $x \theta$ and Lemma 4.3, there exists $c$ such that $x \theta_{\mid v}=c$ and $c \rightarrow^{*} t_{\mid v}$, so that $\Phi_{1}$ can do a transformation

$$
\Gamma\left(=\Gamma^{\prime} \cup\{p\}\right) \Rightarrow_{\Phi_{1}} \Gamma^{\prime} \cup\left\{x \approx s[c]_{v}, c \approx s_{\mid v}\right\}(=\tilde{\Gamma})
$$

(or if $s[c]_{v} \in G$ then

$$
\left.\Gamma\left(=\Gamma^{\prime} \cup\{p\}\right) \Rightarrow_{\Phi_{1}} \Gamma^{\prime} \cup\left\{x \approx_{\mathrm{vf}} s[c]_{v}, c \approx s_{\mid v}\right\}(=\tilde{\Gamma})\right)
$$

and $\theta$ is also a locally minimum $R$-unifier of $\tilde{\Gamma}$. Thus, (V1) holds.

Conversely, if $\theta^{\prime}$ is an $R$-unifier of $\operatorname{Core}(\tilde{\Gamma})$, then $x \theta^{\prime} \leftrightarrow^{*} s[c]_{v} \theta^{\prime}$ and $c \leftrightarrow^{*} s_{\mid v} \theta^{\prime}$. So there exists a sequence $x \theta^{\prime} \leftrightarrow^{*} s \theta^{\prime}$, i.e., $\theta^{\prime}$ is an $R$-unifier of Core $(\{p\})$. So, (V2) holds.

Next, we consider the case of $p=x \triangleright s$. Since $\gamma$ :

$x \theta \rightarrow \rightarrow^{*} r \stackrel{\geq O_{X}(s)}{\leftrightarrow}{ }^{*} s \theta$ and there exists $v \in \operatorname{Min}(\gamma)$ such that $v<u$ for some $u \in O_{X}(s)$, there exist sequences $\gamma^{\prime}: s \theta \stackrel{\geq O_{X}(s)}{\rightarrow} t$ and $\gamma^{\prime \prime}: x \theta \rightarrow^{*} r \stackrel{\geq O_{X}(s)}{\rightarrow}$ ( $t$ for some $t$ such that $v \in \operatorname{Min}\left(\gamma^{\prime \prime}\right)$. By minimum of $x \theta$ and Lemma 4.3, there exists $c$ such that $x \theta_{\mid v}=c$ and $c \rightarrow^{*} r_{\mid v} \stackrel{\geq O_{X}\left(s_{\mid v}\right)}{\rightarrow} t^{*}$. By $\gamma^{\prime}$ and $s_{\mid v} \in S$, we have $s_{\mid v} \theta \stackrel{\geq O_{X}\left(s_{v}\right)}{\rightarrow} t_{\mid v}$, so that $\Phi_{1}$ can do a transformation

$$
\Gamma\left(=\Gamma^{\prime} \cup\{p\}\right) \Rightarrow_{\Phi_{1}} \Gamma^{\prime} \cup\left\{x \triangleright s[c]_{v}, c \triangleright s_{\mid v}\right\}(=\tilde{\Gamma})
$$

(or if $s[c]_{v} \in G$ then

$$
\left.\Gamma\left(=\Gamma^{\prime} \cup\{p\}\right) \Rightarrow_{\Phi_{1}} \Gamma^{\prime} \cup\left\{x \approx_{\mathrm{vf}} s[c]_{v}, c \triangleright s_{\mid v}\right\}(=\tilde{\Gamma})\right)
$$

and $\theta$ is also a locally minimum $R$-unifier of $\tilde{\Gamma}$. Thus, (V1) holds.

Conversely, if $\theta^{\prime}$ is an $R$-unifier of $\operatorname{Core}(\tilde{\Gamma})$, then there exist sequences $\gamma^{\prime}: x \theta^{\prime} \leftrightarrow^{*} s[c]_{v} \theta^{\prime}$ and $\gamma^{\prime \prime}: c \leftrightarrow^{*} s_{\mid v} \theta^{\prime}$. So there exists a sequence $x \theta^{\prime} \leftrightarrow^{*} s \theta^{\prime}$, i.e., $\theta^{\prime}$ is an $R$-unifier of Core $(\{p\})$. So, (V2) holds.

We have proved this lemma for all the cases of $\Gamma$, so this lemma holds.

\subsection{Correctness of Stage II}

Let $E_{2}=\left\{s \approx_{\mathrm{vf}} t \mid s \notin S\right\}$. Note that for the Conversion $\Gamma \Rightarrow_{\Phi_{1}} \operatorname{Conv}(\Gamma)$ in Stage I, we have $\operatorname{Conv}(\Gamma) \subseteq E_{2}$, and for every transformation $\Gamma \Rightarrow_{\Phi_{2}} \tilde{\Gamma}$ in Stage II, $\Gamma \subseteq E_{2}$ implies $\tilde{\Gamma} \subseteq E_{2}$. The proof is straightforward, so omitted.

Lemma 6.4: Stage II is terminating and finite-branching.

Proof For $\Gamma \subseteq E_{2}$, we define size $(\Gamma)=\left(\$_{1}(\Gamma), \$_{2}(\Gamma)\right)$.

Here

$$
\begin{aligned}
& \$_{1}(\Gamma)=\sqcup_{s \approx_{\mathrm{vf}} t \in \Gamma}\left(\operatorname{HD}_{G}(s) \sqcup \mathrm{HD}_{G}(t)\right) \\
& \$_{2}(\Gamma)=|\Gamma|
\end{aligned}
$$

We use the lexicographic ordering $>_{\text {size }}$ to compare any $\Gamma, \Gamma^{\prime} \subseteq E_{2}$.

For every transformation $\Phi_{2}(\Gamma)=\left\{\Gamma_{1}, \cdots, \Gamma_{k}\right\}$ in Stage II, we prove that $\Gamma>_{\text {size }} \Gamma_{i}$ for every $i \in\{1, \cdots, k\}$ by verifying the following table. 


\begin{tabular}{l|ll} 
& $\$_{1}$ & $\$_{2}$ \\
\hline Decomposition, Substitution & $\gg$ & \\
GT & $\gg$ & $>$
\end{tabular}

Let $\Gamma \Rightarrow_{\Phi_{2}} \tilde{\Gamma}$ and $\Gamma^{\prime}=\Gamma \backslash\{p\}$.

\section{Decomposition}

Let $p=x \approx_{\mathrm{vf}} s$ and $q=x \approx_{\mathrm{vf}} t$ be such that $s \neq t, s, t \in$ $S$, common $\left(s^{\prime}, t^{\prime}\right)$, and $s^{\prime} \gg_{\mathrm{HD}_{G}} t^{\prime}$, where $s^{\prime}=\operatorname{gmin}(s, U \cup V)$, $t^{\prime}=\operatorname{gmin}(t, U \cup V), \bar{U}=\operatorname{Min}\left(O_{X}(s) \cup O_{X}(t)\right), V=$ $\operatorname{Min}\left(O_{G}(s) \cap O_{G}(t)\right)$. Then Decomposition replaces $\{p\}$ by $\left\{s_{\mid u}^{\prime} \approx_{\mathrm{vf}} t_{\mid u}^{\prime} \mid u \in U\right.$ and $\left.s_{\mid u}^{\prime} \in X\right\} \cup\left\{t_{\mid u}^{\prime} \approx_{\mathrm{vf}} s_{\mid u}^{\prime} \mid u \in U\right.$ and $s_{\mid u}^{\prime} \notin$ $X\}$ and $\{q\}$ by $\left\{x \approx_{\mathrm{vf}} t^{\prime}\right\}\left(=\left\{q^{\prime}\right\}\right)$, respectively. Here, $t=\mathrm{HD}_{G} t^{\prime}$ and $s=\mathrm{HD}_{G} s^{\prime}$ hold, since only ground subterms are replaced by other ground terms. Since $s^{\prime} \gg \mathrm{HD}_{G} s_{\mid u}^{\prime}$ and $s^{\prime} \gg_{\mathrm{HD}_{G}} t^{\prime} \gg_{\mathrm{HD}_{G}} t_{\mid u}^{\prime}$ holds for every $u \in U$ by Lemma 6.1 (1), the $\$_{1}$-value strictly decreases.

\section{Substitution}

If $p=x \approx_{\mathrm{vf}} s$ or $s \approx_{\mathrm{vf}} x$ is such that $s \notin S$, then Substitution replaces $\Gamma^{\prime} \cup\{p\}$ by $\Gamma^{\prime} \sigma$ such that $\sigma=\left\{x \rightarrow s^{\prime}\right\}$ and $s^{\prime}$ is the minimum term in $\mathcal{L}(s)$. By Lemma $6.1(3), \Gamma^{\prime} \geqq_{\$_{1}} \Gamma^{\prime} \sigma$ holds. Thus, $\$_{1}\left(\Gamma^{\prime} \cup\{p\}\right)=\$_{1}\left(\Gamma^{\prime}\right) \sqcup \mathrm{HD}_{G}(x) \sqcup \mathrm{HD}_{G}\left(s^{\prime}\right) \gg \$_{1}\left(\Gamma^{\prime} \sigma\right)$ holds, so that the $\$ 1$-value strictly decreases.

\section{GT Transformation}

Let $p=s \approx_{\mathrm{vf}} t$ be such that $s \in G, t \notin X$ and common $\left(s^{\prime}, t^{\prime}\right)$, where $s^{\prime}=\operatorname{gmin}(s, U \cup V), t^{\prime}=\operatorname{gmin}(t, U \cup V), U=O_{X}(t)$, $V=\operatorname{Min}\left(O_{G}(t)\right)$. Then the GT transformation replaces $\{p\}$ by $\left\{t_{\mid u}^{\prime} \approx_{\mathrm{vf}} s_{\mid u}^{\prime} \mid u \in U\right\}$. If $t^{\prime} \in S$ then $\$_{1}(\{p\})=$ $\mathrm{HD}_{G}\left(t^{\prime}\right) \gg \mathrm{HD}_{G}\left(t_{\mid u}^{\prime}\right)=\$_{1}\left(\left\{t_{\mid u}^{\prime} \approx_{\mathrm{vf}} s_{\mid u}^{\prime}\right\}\right)$ for every $u \in O_{X}\left(t^{\prime}\right)$ by Lemma 6.1(1) and $\mathrm{HD}_{G}\left(s_{\mid u}^{\prime}\right)=\emptyset$, so that the $\$_{1}$-value strictly decreases. If $t^{\prime} \in G$ then $\left\{t_{\mid u}^{\prime} \approx_{\mathrm{vf}} s_{\mid u}^{\prime} \mid u \in U\right\}=\emptyset$, so that the $\$_{1}$-value is unchanged and the $\$_{2}$-value strictly decreases.

Moreover, if $\Gamma$ is a finite set, then $k$ is finite, i.e., Stage II is finite branching. Thus, this lemma holds.

\section{Lemma 6.5:}

(i) Stage II is valid.

(ii) If $\Gamma \subseteq E_{2}$ is $R$-unifiable and does not satisfy the stop condition of Stage II, then $\Phi_{2}(\Gamma) \neq \emptyset$.

Proof We first show that $\Phi_{2}$ satisfies (ii) of Lemma 6.5. For $\Gamma \subseteq E_{2}$, if $\Gamma$ contains $p=x \approx_{\mathrm{vf}} s$ or $s \approx_{\mathrm{vf}} x$ with $s \notin S$ then we can obviously do Substitution, and if $\Gamma$ contains $p=s \approx_{\mathrm{vf}} t$ with $s \in G, t \notin X$, then we can do the GT transformation since common $\left(s^{\prime}, t^{\prime}\right)$ where $s^{\prime}=\operatorname{gmin}(s, U \cup V)$, $t^{\prime}=\operatorname{gmin}(t, U \cup V), U=O_{X}(t), V=\operatorname{Min}\left(O_{G}(t)\right)$ by $R$ unifiability of $\Gamma$. Thus, the remaining case is that $\Gamma \subseteq$ $\left\{x \approx_{\mathrm{vf}} t \mid t \in S\right\}$. In this case, if $\Gamma$ does not satisfy the stop condition of Stage II, i.e., $\Gamma$ is not in solved form, we can do Decomposition since common $\left(s^{\prime}, t^{\prime}\right)$ where $s^{\prime}=$ $\operatorname{gmin}(s, U \cup V), t^{\prime}=\operatorname{gmin}(t, U \cup V), U=\operatorname{Min}\left(O_{X}(s) \cup O_{X}(t)\right)$,
$V=\operatorname{Min}\left(O_{G}(s) \cap O_{G}(t)\right)$ by the $R$-unifiability of $\Gamma$ as we will prove later. Thus, (ii) of Lemma 6.5 holds.

Next we show that every transformation in Stage II satisfies the validity conditions (V1) and (V2). To show (V1), we assume that $\theta$ is a locally minimum $R$-unifier of $\Gamma$ and $\Gamma \Rightarrow_{\Phi_{2}} \tilde{\Gamma}$.

\section{Decomposition}

Let $p, q \in \Gamma, p=x \approx_{\mathrm{vf}} s$ and $q=x \approx_{\mathrm{vf}} t$ be such that $s, t \in S$ and $s \neq t$. Since $\theta$ is a locally minimum $R$-unifier of $p$, there exist sequences $\gamma_{x s}: x \theta \leftrightarrow^{*} s \theta$, where $\gamma_{x s}$ is $O_{X}(s)$-frontier. Let $s^{\prime}=\operatorname{gmin}(s, U \cup V)$, where $U=\operatorname{Min}\left(O_{X}(s) \cup O_{X}(t)\right)$, $V=\operatorname{Min}\left(O_{G}(s) \cap O_{G}(t)\right)$, then there exists a sequence $\gamma_{s s^{\prime}}: s \theta \leftrightarrow^{*} s^{\prime} \theta$, where $\gamma_{s s^{\prime}}$ is $O_{X}(s)\left(=O_{X}\left(s^{\prime}\right)\right)$-frontier. Thus, $x \theta_{\mid w} \leftrightarrow^{*} s_{\mid w}^{\prime}$ for any $w \in \operatorname{Min}\left(O_{G}\left(s^{\prime}\right)\right)$. By Lemma 4.2 and the definition of $s^{\prime}, x \theta_{\mid w}$ and $s_{\mid w}^{\prime}$ are minimum, so $x \theta_{\mid w}=$ $s_{\mid w}^{\prime}$. Thus, $x \theta \stackrel{\geq O_{X}\left(s^{\prime}\right)}{\leftrightarrow} s^{\prime} \theta$. Since $\theta$ is also a locally minimum $R$-unifier of $q, x \theta \stackrel{\geq O_{X}\left(t^{\prime}\right)}{\leftrightarrow} t^{*} \theta$ where $t^{\prime}=\operatorname{gmin}(t, U \cup V)$. So, $s^{\prime} \theta \stackrel{\geq U}{\leftrightarrow} t^{\prime} \theta$ holds. Thus, common $\left(s^{\prime}, t^{\prime}\right)$ and $\Phi_{2}$ can do a Decomposition transformation

$$
\begin{aligned}
& \Gamma\left(=\Gamma^{\prime} \cup\{p, q\}\right) \\
& \Rightarrow_{\Phi_{2}} \Gamma^{\prime} \cup\left\{q^{\prime}\right\} \\
& \cup\left\{s_{\mid u}^{\prime} \approx_{\mathrm{vf}} t_{\mid u}^{\prime} \mid u \in U \text { and } s_{\mid u}^{\prime} \in X\right\} \\
& \cup\left\{t_{\mid u}^{\prime} \approx_{\mathrm{vf}} s_{\mid u}^{\prime} \mid u \in U \text { and } s_{\mid u}^{\prime} \notin X\right\}(=\tilde{\Gamma})
\end{aligned}
$$

where $q^{\prime}=x \approx_{\mathrm{vf}} t^{\prime}$. For any $u \in U, s_{\mid u}^{\prime} \theta \stackrel{\geq O_{X}\left(s_{\mid u}^{\prime}\right)}{\leftrightarrow} \leftrightarrow^{*} x \theta_{\mid u} \stackrel{\geq O_{X}\left(t_{l_{u}^{\prime}}^{\prime}\right)}{\leftrightarrow}$ $t_{\mid u}^{\prime} \theta$. By Lemma 4.2, $x \theta_{\mid u}$ is minimum. If $s_{\mid u}^{\prime} \in X$ then $s_{\mid u}^{\prime} \theta=x \theta_{\mid u}$ since $s_{\mid u}^{\prime} \theta$ is minimum. Thus, $\theta$ is also a locally minimum $R$-unifier of $s_{\mid u}^{\prime} \approx_{\mathrm{vf}} t_{\mid u}^{\prime}$. Similarly, if $s_{\mid u}^{\prime} \notin X$ then $\theta$ is a locally minimum $R$-unifier of $t_{\mid u}^{\prime} \approx_{\mathrm{vf}} s_{\mid u}^{\prime}$. Thus, the validity condition (V1) holds.

Conversely, let $\theta^{\prime}$ be an $R$-unifier of Core $(\tilde{\Gamma})$. It suffices to prove that $\theta^{\prime}$ is an $R$-unifier of $x \approx s$. Since $\theta^{\prime}$ is an $R$ unifier of $\operatorname{Core}(\tilde{\Gamma})$, for any $u \in U, s_{\mid u}^{\prime} \theta^{\prime} \leftrightarrow^{*} t_{\mid u}^{\prime} \theta^{\prime}$ holds, and common $\left(s^{\prime}, t^{\prime}\right)$ so that $s^{\prime} \theta^{\prime} \leftrightarrow^{*} t^{\prime} \theta^{\prime}$ and $x \theta^{\prime} \stackrel{\leftrightarrow}{\leftrightarrow}{ }^{*} t \theta^{\prime}$. Thus, $x \theta^{\prime} \leftrightarrow^{*} t \theta^{\prime} \leftrightarrow^{*} t^{\prime} \theta^{\prime} \leftrightarrow^{*} s^{\prime} \theta^{\prime} \leftrightarrow^{*} s \theta^{\prime}$. So, $\theta^{\prime}$ is an $R$-unifier of $x \approx s$. Thus, (V2) holds.

\section{Substitution}

Let $p=x \approx_{\mathrm{vf}} s$, where $s \notin S$. Let $s^{\prime}$ be the minimum term in $\mathcal{L}(s)$. Since $\theta$ is a locally minimum $R$-unifier of $x \approx_{\mathrm{vf}} s$, $x \theta=s^{\prime} \theta$ holds. Thus, $\Phi_{1}$ can do a transformation

$$
\Gamma\left(=\Gamma^{\prime} \cup\{p\}\right) \Rightarrow_{\Phi_{1}} \Gamma^{\prime} \sigma(=\tilde{\Gamma})
$$

where $\sigma=\left\{x \rightarrow s^{\prime}\right\}$. For any $t \approx_{\mathrm{vf}} r \in \Gamma^{\prime}$, there exists a sequence $\gamma: t \sigma \theta \leftrightarrow^{*} r \sigma \theta$, where $\gamma$ is $O_{X}(r)$-frontier, so that $\theta$ is a locally minimum $R$-unifier of $t \sigma \approx_{\mathrm{vf}} r \sigma$. Thus, (V1) holds.

Conversely, let $\theta^{\prime}$ be an $R$-unifier of Core $\left(\Gamma^{\prime} \sigma\right)$, and $\theta^{\prime \prime}$ be a substitution such that $x \theta^{\prime \prime}=s^{\prime} \theta^{\prime}$ and for any $y \in X \backslash\{x\}$, 
$y \theta^{\prime \prime}=y \theta^{\prime}$. For any $t \approx r \in \operatorname{Core}\left(\Gamma^{\prime}\right), t \theta^{\prime \prime}=t \sigma \theta^{\prime} \leftrightarrow^{*} r \sigma \theta^{\prime}=$ $r \theta^{\prime \prime}$ holds. Since $s^{\prime} \in \mathcal{L}(s), x \theta^{\prime \prime}=s^{\prime} \theta^{\prime} \leftrightarrow^{*} s \theta^{\prime}$ holds. Thus, $\theta^{\prime \prime}$ is an $R$-unifier of Core $\left(\Gamma^{\prime} \cup\{p\}\right)$. So, (V2) holds.

GT Transformation

Let $p=s \approx_{\mathrm{vf}} t \in \Gamma$, where $s \in G$ and $t \notin X$. Note that since $\theta$ is a locally minimum $R$-unifier of $p$, there exists a sequence $\gamma: s \leftrightarrow^{*} t \theta$, where $\gamma$ is $U$-frontier and $U=O_{X}(t)$. Let $s^{\prime}=\operatorname{gmin}(s, U \cup V)$ and $t^{\prime}=\operatorname{gmin}(t, U \cup V)$, where $V=\operatorname{Min}\left(O_{G}(t)\right)$, then there exist sequences $\gamma_{s s^{\prime}}: s \leftrightarrow^{*} s^{\prime}$ and $\gamma_{t t^{\prime}}: t \theta \leftrightarrow^{*} t^{\prime} \theta$, where $\gamma_{t t^{\prime}}$ is $U\left(=O_{X}\left(t^{\prime}\right)\right)$-frontier. Thus, $s_{\mid v}^{\prime} \leftrightarrow^{*} t_{\mid v}^{\prime}$ for any $v \in V$. By the definition of $s^{\prime}$ and $t^{\prime}, s_{\mid v}^{\prime}$ and $t_{\mid v}^{\prime}$ are minimum, so $s_{\mid v}^{\prime}=t_{\mid v}^{\prime}$. Then, $s^{\prime} \stackrel{\geq U}{\leftrightarrow} t^{\prime} \theta$. Thus, common $\left(s^{\prime}, t^{\prime}\right)$ and $\Phi_{2}$ can do a GT transformation

$$
\Gamma\left(=\Gamma^{\prime} \cup\{p\}\right) \Rightarrow_{\Phi_{2}} \Gamma^{\prime} \cup\left\{t_{\mid u}^{\prime} \approx_{\mathrm{vf}} s_{\mid u}^{\prime} \mid u \in U\right\}(=\tilde{\Gamma})
$$

and $\theta$ is also a locally minimum $R$-unifier of $\tilde{\Gamma}$. So, the validity condition (V1) holds.

Conversely, if $\theta^{\prime}$ is an $R$-unifier of $\operatorname{Core}(\tilde{\Gamma})$, then there exist sequences $\gamma_{u}: s_{\mid u}^{\prime} \leftrightarrow^{*} t_{\mid u}^{\prime} \theta^{\prime}$ for any $u \in U$. Since common $\left(s^{\prime}, t^{\prime}\right)$, there exists a sequence $s^{\prime} \leftrightarrow^{*} t^{\prime} \theta^{\prime}$. By $s \leftrightarrow^{*} s^{\prime}$ and $t \theta^{\prime} \leftrightarrow^{*} t^{\prime} \theta^{\prime}, \theta^{\prime}$ is an $R$-unifier of Core $(\{p\})$. So, (V2) holds.

\subsection{Correctness of Final Stage}

Lemma 6.6: Assume that $\Gamma$ satisfies the stop condition of Stage II. Then $\Gamma$ is not cyclic if there exists a locally minimum $R$-unifier $\theta$ of $\Gamma$.

Proof Let $\theta$ be a locally minimum $R$-unifier of $\Gamma$. We first show that for any $x \approx_{\mathrm{vf}} s \in \Gamma$ and $y \in V(s)$, if $s \notin X$ then $x \theta>_{\text {height }} y \theta$. Let $y=s_{\mid u}$ for some $u \neq \varepsilon$. Then $x \theta_{\mid u} \leftrightarrow^{*} y \theta$ holds, since $\theta$ is an $R$-unifier of $\Gamma$. The local minimum of $\theta$ ensures that $x \theta_{\mid u} \geq_{\text {height }} y \theta$. Hence, $x \theta>_{\text {height }} y \theta$. It follows that for any $x, y \in X$, if $x \mapsto_{\Gamma} y$, then $x \theta>_{\text {height }} y \theta$ holds. Therefore, it is impossible that we have $x \mapsto_{\Gamma}^{+} x$. Hence $\Gamma$ is not cyclic.

Lemma 6.7: If $\Gamma$ satisfies the stop condition of Stage II and there exists a locally minimum $R$-unifier of $\Gamma$, then $\Gamma$ is $\emptyset$-unifiable.

Proof Obviously, $\Gamma \neq$ ffail $\}$, so that $\Gamma$ is in solved form. By Lemma $6.6, \Gamma$ is not cyclic and hence $\Gamma$ is $\emptyset$-unifiable.

\subsection{Main Theorem}

Now, we can deduce our main theorem.

Theorem 6.8: The unification problem for confluent semi-constructor TRSs is decidable.

Proof By Lemmata 6.2 and 6.4, part (1) of the correctness condition of $\Phi$ holds and by Lemmata 6.3 and 6.5, Stages I and II are valid, so that if $\Gamma_{0}=\left\{s_{0} \approx t_{0}\right\}$ is $R$-unifiable, then there exist $\Gamma_{1}$ and $\Gamma_{\mathrm{f}}$ such that $\Gamma_{0} \Rightarrow_{\Phi_{1}}^{*} \Gamma_{1} \Rightarrow_{\Phi_{2}}^{*} \Gamma_{\mathrm{f}}, \Gamma_{1}$ satisfies the stop condition of Stage I, $\Gamma_{\mathrm{f}}$ satisfies the one of Stage II, and there exists a locally minimum $R$-unifier of $\Gamma_{\mathrm{f}}$. Hence, by Lemma 6.7, the only-if-part of part (2) of the correctness condition of $\Phi$ holds. Conversely, the ifpart is ensured by validity of the transformations of $\Phi_{1}$ and $\Phi_{2}$. Thus, part (2) of the correctness condition of $\Phi$ holds. Therefore, the theorem follows from the decidability of $\emptyset$ unifiability.

\section{Application of Main Theorem}

In this section, we give a sufficient condition for ensuring the decidability of the unification problem for a new subclass of nonlinear TRSs using our main theorem in the previous section. For example, $R=\{\mathrm{c} \rightarrow \mathrm{g}(\mathrm{c}, \mathrm{c}), \mathrm{g}(x, x) \rightarrow$ $\mathrm{f}(x, \mathrm{~g}(x, \mathrm{~h}(x))), \mathrm{f}(x, x) \rightarrow \mathrm{a}\}$ is not a semi-constructor TRS since the second rule is not. Furthermore, $R$ is not shallow, semi-linear, or linear standard. Here, we introduce a new function symbol $\mathrm{f}_{1}$ and divide the rule as follows: $R^{\prime}=\left\{\mathrm{c} \rightarrow \mathrm{g}(\mathrm{c}, \mathrm{c}), \mathrm{g}(x, x) \rightarrow \mathrm{f}_{1}(x), \mathrm{f}(x, \mathrm{~g}(x, \mathrm{~h}(x))) \rightarrow\right.$ $\left.\mathrm{f}_{1}(x), \mathrm{f}(x, x) \rightarrow \mathrm{a}\right\}$. TRS $R^{\prime}$ is a semi-constructor and we can show that $R^{\prime}$ is confluent, so that the unification problem is decidable for $R^{\prime}$. Moreover, we can show that two terms are $R^{\prime}$-unifiable iff they are $R$-unifiable. Now, we formalize this approach.

Definition 7.1: Let $R$ be a non-semi-constructor TRS and $R_{\mathrm{nsc}}=\{\alpha \rightarrow \beta \in R \mid \alpha \rightarrow \beta$ is not semi-constructor $\}=$ $\left\{\alpha_{i} \rightarrow \beta_{i} \mid 1 \leq i \leq m\right\}$. For each $\alpha_{i} \rightarrow \beta_{i} \in R_{\mathrm{nsc}}$, let $U_{i}=$ $\operatorname{Min}\left\{u \in O\left(\beta_{i}\right) \backslash O_{G}\left(\beta_{i}\right) \mid \operatorname{root}\left(\beta_{i \mid u}\right) \in D_{R}\right\}=\left\{u_{i 1}, \cdots, u_{i k_{i}}\right\}$. Note that $U_{i} \neq \emptyset$. Let $F_{i}^{\prime}=\left\{f_{i 1}, \cdots, f_{i k_{i}}\left|k_{i}=\right| U_{i} \mid\right\}$ and $F^{\prime}=\bigcup_{1 \leq i \leq m} F_{i}^{\prime}$, where $F \cap F^{\prime}=\emptyset$. Let $t_{i j}=f_{i j}\left(x_{1}, \cdots, x_{l}\right)$, $f_{i j} \in F_{i}^{\prime}$ where $\mathrm{V}\left(\beta_{i \mid u_{i j}}\right)=\left\{x_{1}, \cdots, x_{l}\right\}$. Then, TRS $\Psi(R)$ is constructed as follows:

$$
\begin{aligned}
& \Psi(R)=\left(R \backslash R_{\mathrm{nsc}}\right) \cup \\
& \bigcup_{1 \leq i \leq m}\left\{\alpha_{i} \rightarrow \beta_{i}\left[t_{i 1}, \cdots, t_{i k_{i}}\right]_{\left(u_{i 1}, \cdots, u_{i k_{i}}\right)},\right. \\
& \left.\beta_{i_{\mid} u_{i j}} \rightarrow t_{i j}\left|k_{i}=\right| U_{i} \mid, 1 \leq j \leq k_{i}\right\}
\end{aligned}
$$

Note that $D_{R}=D_{\Psi(R)}$ and $D_{\Psi(R)} \cap F^{\prime}=\emptyset$, so that $\Psi(R)$ is a semi-constructor TRS.

We define $\phi: T \rightarrow T$ as follows.

$$
\phi(t)=\left\{\begin{array}{l}
\beta_{i \mid u_{i j} \sigma_{i j}} \\
\left(\text { if } t=f_{i j}\left(t_{1}, \cdots, t_{l}\right), f_{i j} \in F^{\prime}\right) \\
f\left(\phi\left(t_{1}\right), \cdots, \phi\left(t_{l}\right)\right) \\
\left(\text { if } t=f\left(t_{1}, \cdots, t_{l}\right), f \in F\right) \\
t(\text { if } t \in X)
\end{array}\right.
$$

Here, $\beta_{i \mid u_{i j}} \rightarrow f_{i j}\left(x_{1}, \cdots, x_{l}\right) \in \Psi(R)$ and $\sigma_{i j}=\left\{x_{k} \rightarrow \phi\left(t_{k}\right) \mid\right.$ $1 \leq k \leq l\}$.

For TRSs $R$ and $\Psi(R)$, the following lemmata hold.

Lemma 7.2: If $s \rightarrow \Psi(R) t$ then $\phi(s) \rightarrow_{R}^{*} \phi(t)$ for every $s, t$. Proof By induction on the structure of $s$.

Basis: Since $s \in X, s \rightarrow \Psi(R) t$ is impossible, so that this lemma holds. 
Induction step: Let $s \stackrel{p}{\rightarrow} \Psi(R) t$.

Case of $p>\varepsilon$ : Let $s=f\left(s_{1}, \cdots, s_{l}\right)$, then $t=$ $f\left(t_{1}, \cdots, t_{l}\right)$ and either $s_{k} \rightarrow \Psi(R) t_{k}$ or $s_{k}=t_{k}$ for every $k \in$ $\{1, \cdots, l\}$. By the induction hypothesis, $\phi\left(s_{k}\right) \rightarrow_{R}^{*} \phi\left(t_{k}\right)$ for every $k \in\{1, \cdots, l\}$. Thus, if $f \in F$ then $\phi(s) \rightarrow_{R}^{*} \phi(t)$ holds. Otherwise, since $f=f_{i j}$ for some $\beta_{i \mid u_{i j}} \rightarrow f_{i j}\left(x_{1}, \cdots, x_{l}\right) \in$ $\Psi(R), \phi(s)=\beta_{i \mid u_{i j}} \sigma$ and $\phi(t)=\beta_{i \mid u_{i j}} \sigma^{\prime}$ where $\sigma=\left\{x_{k} \rightarrow\right.$ $\left.\phi\left(s_{k}\right) \mid 1 \leq k \leq l\right\}$ and $\sigma^{\prime}=\left\{x_{k} \rightarrow \phi\left(t_{k}\right) \mid 1 \leq k \leq l\right\}$. Thus, $\phi(s) \rightarrow_{R}^{*} \phi(t)$ holds.

Case of $p=\varepsilon$ : Let $s=\alpha \theta \rightarrow \Psi(R) \beta \theta=t$ where $\alpha \rightarrow \beta$ is a rewrite rule. Obviously, $\alpha \theta^{\prime} \rightarrow \Psi(R) \beta \theta^{\prime}$ holds for $\theta^{\prime}=$ $\{x \rightarrow \phi(r) \mid x \rightarrow r \in \theta\}$. If $\alpha \rightarrow \beta \in R$ then $\phi(s)=$ $\alpha \theta^{\prime} \rightarrow_{R} \beta \theta^{\prime}=\phi(t)$ holds. Otherwise, if $\alpha=\alpha_{i}$ for some $i \in\{1, \cdots, n\}$ then $\beta=\beta_{i}\left[t_{i 1}, \cdots, t_{i k}\right]_{\left(u_{i 1}, \cdots, u_{i k}\right)}$. Here, $\phi(t)=$ $\phi\left(\beta_{i}\left[t_{i 1}, \cdots, t_{i k}\right]_{\left(u_{i 1}, \cdots, u_{i k}\right)} \theta\right)=\beta_{i} \theta^{\prime}$ by the definition of $\phi$, so that $\phi(s)=\alpha_{i} \theta^{\prime} \rightarrow_{R} \beta_{i} \theta^{\prime}=\phi(t)$ holds. If $\alpha=\beta_{i \mid u_{i j}}$ for some $i \in\{1, \cdots, m\}$ and $j \in\{1, \cdots, k\}$ then $\beta=f_{i j}\left(x_{1}, \cdots, x_{l}\right)$, so that $\phi(s)=\beta_{i \mid u_{i j}} \theta^{\prime}=\phi(t)$ holds.

Lemma 7.3: For any $s, t$ which do not contain function symbols in $F^{\prime}, s$ and $t$ are unifiable for $R$ iff $s$ and $t$ are unifiable for $\Psi(R)$.

Proof If part: By Lemma 7.2. Only if part: Obvious.

By Lemma 7.3, we can deduce the following theorem.

Theorem 7.4: Let $C=\{R \mid \operatorname{TRS} \Psi(R)$ is confluent $\}$. Then, the unification problem for $C$ is decidable.

It is known that strongly weight-preserving and nonE-overlapping (or root-E-closed) TRSs are confluent [4]. We can easily show that every semi-constructor TRS is strongly weight-preserving, so that we can obtain the following corollary.

Corollary 7.5: Let $C^{\prime}=\{R \mid$ TRS $\Psi(R)$ is non $-\mathrm{E}-$ overlapping or root-E-closed $\}$. Then, the unification problem for $C^{\prime}$ is decidable.

\section{Conclusion}

In this paper, we have shown that the unification problem is decidable for semi-constructor TRSs by assuming the confluence as our main theorem. Moreover, we give a sufficient condition for ensuring the decidability of the unification problem for a new subclass of nonlinear TRSs using our main theorem.

\section{References}

[1] F. Baader and T. Nipkow, Term Rewriting and All That, Cambridge University Press, 1998.

[2] H. Comon, M. Haberstrau, and J.-P. Jouannaud, "Syntacticness, cycle-syntacticness and shallow theories," Inf. Comput., vol.111, no.1, pp.154-191, 1994.

[3] N. Dershowitz and J.-P. Jouannaud, "Rewrite systems," in Handbook of Theoretical Computer Science, ed. J. van Leeuwen, vol.B, pp.243-320, Elsevier Science Publishers B.V., 1990.

[4] H. Gomi, M. Oyamaguchi, and Y. Ohta, "On the church-rosser property of root-E-overlapping and strongly depth-preserving term rewriting systems," Trans. IPS Japan, vol.39, no.4, pp.992-1005, 1998.
[5] J.-M. Hullot, "Canonical forms and unification," Proc. 5th Conf. on Automated Deduction, pp.318-334, LNCS 87, 1980.

[6] F. Jacquemard, C. Meyer, and C. Weidenbach, "Unification in extensions of shallow equational theories," Proc. 9th RTA, LNCS 1379, pp.76-90, 1998.

[7] A. Martelli and G. Rossi, "Efficient unification with infinite terms in logic programming," Proc. 5th Generation Computer Systems, pp.202-209, 1984.

[8] I. Mitsuhashi, Decision Problems for Non-Linear Term Rewriting Systems, PhD thesis, Graduate School of Engineering, Mie University, 2006.

[9] I. Mitsuhashi, M. Oyamaguchi, Y. Ohta, and T. Yamada, "On the unification problem for confluent monadic term rewriting systems," Trans. IPS Japan on Programming, 44 (SIG 4 (PRO 17)), pp.54-66, 2003.

[10] I. Mitsuhashi, M. Oyamaguchi, Y. Ohta, and T. Yamada, "The joinability and related decision problems for semi-constructor TRSs," Trans. IPS Japan, vol.47, no.5, pp.1502-1514, 2005.

[11] I. Mitsuhashi, M. Oyamaguchi, and T. Yamada, "The reachability and related decision problems for monadic and confluent semiconstructor TRSs," Inf. Process. Lett., vol.98, pp.219-224, 2005.

[12] R. Nieuwenhuis, "Basic paramodulation and decidable theories," Proc. 11th IEEE Symp. Logic in Computer Science, pp.473-482, 1996.

[13] M. Oyamaguchi, "On the word problem for right-ground termrewriting systems," IEICE Trans., vol.E73, no.5, pp.718-723, May 1990.

[14] M. Oyamaguchi and Y. Ohta, "The unification problem for confluent right-ground term rewriting systems," Inf. Comput., vol.183, no.2, pp.187-211, 2003.

[15] K. Salomaa, "Deterministic tree pushdown automata and monadic tree rewriting systems," J. Comput. Syst. Sci., vol.37, pp.367-394, 1988.

[16] Terese, Term Rewriting Systems, Cambridge University Press, 2003.

\section{Appendix A: Function S}

Let $R_{0}$ be a confluent semi-constructor TRS. The corresponding standard TRS is constructed as follows. The construction has a loop structure. We use $k$ as the loop counter. First, we choose $\alpha \rightarrow \beta \in R_{k}(k \geq 0)$ that does not satisfy the standardness condition. If $\alpha \in F_{0}$ then let $\left\{u_{1}, \cdots, u_{m}\right\}$ be $\{1, \cdots, \operatorname{ar}(\operatorname{root}(\beta))\} \backslash O_{F_{0}}(\beta)$. Otherwise, let $\left\{u_{1}, \cdots, u_{m}\right\}$ be $\operatorname{Min}\left(O_{G}(\beta)\right) \backslash O_{F_{0}}(\beta)$. Let $R_{k+1}=\left(R_{k} \backslash\{\alpha \rightarrow \beta\}\right) \cup\{\alpha \rightarrow$ $\left.\beta\left[d_{1}, \cdots, d_{m}\right]_{\left(u_{1}, \cdots, u_{m}\right)}\right\} \cup\left\{d_{i} \rightarrow \beta_{\mid u_{i}} \mid 1 \leq i \leq m\right\}$ where $d_{1}, \cdots, d_{m}$ are new pairwise distinct constants which do not appear in $R_{k}$ or $T$. This procedure is applied repeatedly until the TRS satisfies the condition of standardness. Let $S$ be this construction procedure and $\mathrm{S}\left(R_{0}\right)$ be the output of $\mathrm{S}$ for input $R_{0}$. It is obvious that $\mathrm{S}$ is terminating.

Example Appendix A.1: $\quad$ Let $R_{0}=\left\{\mathrm{f}_{1}(x) \rightarrow \mathrm{g}(x, \mathrm{~g}(\mathrm{a}, \mathrm{b}))\right.$, $\left.\mathrm{f}_{2}(x) \rightarrow \mathrm{f}_{2}(\mathrm{~g}(\mathrm{c}, \mathrm{d}))\right\}$. Since either $\mathrm{f}_{1}(x)$ and $\mathrm{f}_{2}(x)$ are not constant symbols, $R_{1}=\left\{\mathrm{f}_{1}(x) \rightarrow \mathrm{g}\left(x, \mathrm{~d}_{1}\right), \mathrm{d}_{1} \rightarrow\right.$ $\left.\mathrm{g}(\mathrm{a}, \mathrm{b}), \mathrm{f}_{2}(x) \rightarrow \mathrm{d}_{2}, \mathrm{~d}_{2} \rightarrow \mathrm{f}_{2}(\mathrm{~g}(\mathrm{c}, \mathrm{d})\}\right)$, where $\mathrm{d}_{1}$ and $\mathrm{d}_{2}$ are new constant symbols. Since $d_{2}$ is a constant symbol, $R_{2}=\left\{\mathrm{f}_{1}(x) \rightarrow \mathrm{g}\left(x, \mathrm{~d}_{1}\right), \mathrm{d}_{1} \rightarrow \mathrm{g}(\mathrm{a}, \mathrm{b}), \mathrm{f}_{2}(x) \rightarrow \mathrm{d}_{2}, \mathrm{~d}_{2} \rightarrow\right.$ $\left.f_{2}\left(d_{3}\right), d_{3} \rightarrow g(c, d)\right\}$, where $d_{3}$ is a new constant symbol. Since $R_{2}$ is standard, $\mathrm{S}\left(R_{0}\right)$ returns $R_{2}$. 


\section{Appendix B: Function M}

\section{Definition Appendix B.1:}

(1) For a term $\alpha$, let $\operatorname{Rhs}(\alpha, R)=\{\beta \mid \alpha \rightarrow \beta \in R\}$.

(2) For $\Delta \subseteq G$, let $\operatorname{Cut}(\Delta)=\left\{(u, d) \mid u \in \operatorname{Min}\left(\cup_{s \in \Delta} O_{F_{0}}(s)\right)\right.$ and $d$ sord $s_{\mid u}$ for every $\left.s \in \Delta\right\}$. (The measure ord is defined in Definition 4.1) For example, $\operatorname{Cut}(\{\neg(\neg(\mathrm{t})), \neg(\mathrm{f})\})=\{(1, \mathrm{f})\}$.

\section{Definition Appendix B.2: Let}

$\operatorname{Rhs}\left(d, R_{\mathrm{C}}\right)=\left\{s_{1}, \cdots, s_{m}\right\}$ and $\operatorname{Cut}\left(\operatorname{Rhs}\left(d, R_{\mathrm{C}}\right)\right)=$ $\left\{\left(u_{1}, d_{1}\right), \cdots,\left(u_{n}, d_{n}\right)\right\}$.

Then we define Normalize $\left(d, R_{\mathrm{C}}\right)=$ $\left\{d \rightarrow s_{1}\left[d_{1}, \cdots, d_{n}\right]_{\left(u_{1}, \cdots, u_{n}\right)}\right\} \cup\left\{d_{j} \rightarrow s_{i \mid u_{j}} \mid 1 \leq i \leq\right.$ $\left.m, 1 \leq j \leq n, d_{j} \neq s_{i \mid u_{j}}\right\}$. For example, Normalize $(\mathrm{t},\{\mathrm{t} \rightarrow$ $\neg(\neg(\mathrm{t})), \mathrm{t} \rightarrow \neg(\mathrm{f})\})=\{\mathrm{t} \rightarrow \neg(\mathrm{f}), \mathrm{f} \rightarrow \neg(\mathrm{t})\}$.

Each of the following functions takes as input a quasistandard confluent and semi-constructor TRS $R$. Note that if $R^{\prime}=\operatorname{Determinize}(R)$ then $\left|\operatorname{Rhs}\left(d, R_{C}^{\prime}\right)\right| \leq 1$ for any $d$ by the termination condition of Determinize. Henceforth, we use $(A \circ B)(x)$ to denote $A(B(x))$ for functions $A, B$.
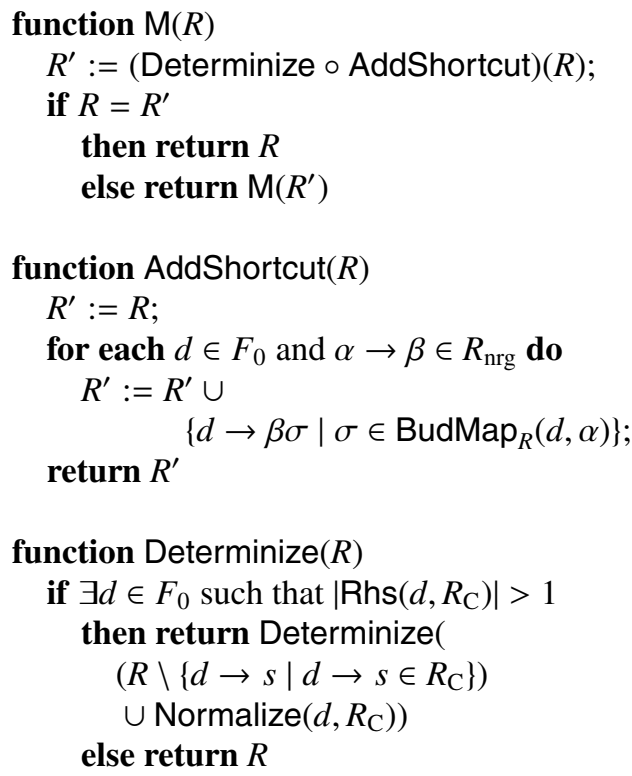

Example Appendix B.3: $\quad$ For TRS $R_{\mathrm{e}}$ of Example 2.2, $\mathrm{M}\left(R_{\mathrm{e}}\right)$ is computed as follows. AddShortcut $\left(R_{\mathrm{e}}\right)$ is first called and a new shortcut rule $\mathrm{t} \rightarrow \neg(\wedge(\mathrm{f}, \mathrm{f}))$ is added to $R_{\mathrm{e}}$ since $\mathrm{t} \rightarrow \operatorname{nand}(\mathrm{f}, \mathrm{f})$, nand $(x, x) \rightarrow \neg(\wedge(x, x)) \in$ $R_{\mathrm{e}}$. By $\mathrm{f} \rightarrow$ nand $(\mathrm{t}, \mathrm{t}) \in R_{\mathrm{e}}, \mathrm{f} \rightarrow \neg(\wedge(\mathrm{t}, \mathrm{t}))$ is also added. Thus, AddShortcut $\left(R_{\mathrm{e}}\right)=R^{\prime}$ where $R^{\prime}=R_{\mathrm{e}} \cup$ $\{\mathrm{t} \rightarrow \neg(\wedge(\mathrm{f}, \mathrm{f})), \mathrm{f} \rightarrow \neg(\wedge(\mathrm{t}, \mathrm{t}))\}$. Next, Determinize $\left(R^{\prime}\right)$ is called and returns the same $R^{\prime}$ as output. Since $R^{\prime} \neq R_{\mathrm{e}}$, (Determinize $\circ$ AddShortcut) $\left(R^{\prime}\right)$ is computed. Note that $R_{\mathrm{C}}^{\prime}=\{\mathrm{t} \rightarrow \neg(\wedge(\mathrm{f}, \mathrm{f})), \mathrm{f} \rightarrow \neg(\wedge(\mathrm{t}, \mathrm{t}))\}$. AddShortcut $\left(R^{\prime}\right)$ returns the same $R^{\prime}$ and so Determinize $\left(R^{\prime}\right)$. Thus, this algorithm halts. $\mathrm{M}\left(R_{\mathrm{e}}\right)$ returns $R^{\prime}$ as output. That is, $\mathrm{M}\left(R_{\mathrm{e}}\right)=$ $R_{\mathrm{e}} \cup\{\mathrm{t} \rightarrow \neg(\wedge(\mathrm{f}, \mathrm{f})), \mathrm{f} \rightarrow \neg(\wedge(\mathrm{t}, \mathrm{t}))\}$.

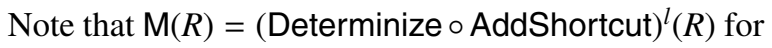

some $l \geq 1, R_{\mathrm{nrg}}=\mathrm{M}(R)_{\mathrm{nrg}}$, and $\mathrm{M}(\mathrm{M}(R))=\mathrm{M}(R)$ when $\mathrm{M}(R)$ halts. In the produced TRS $\mathrm{M}(R)$, the heights of some right-hand side terms of type $\mathrm{C}$ rules may become greater than 1.

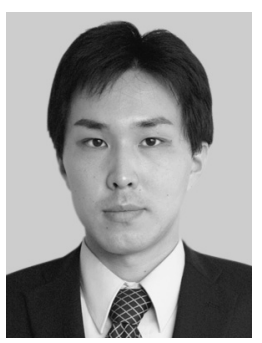

Ichiro Mitsuhashi was born in 1978. He received the Dr. Eng. degree from Mie University in 2006. He is now an Assistant Professor of the Center for Information Technologies and Networks of Mie University. His current research interest is term rewriting systems.

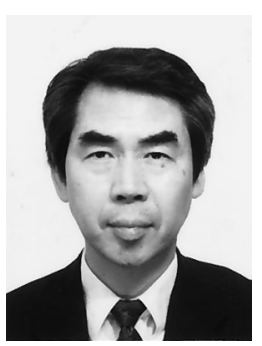

Michio Oyamaguchi was born in 1947. He received the Dr. Eng. degree from Tohoku University in 1977. He is now a Professor of the Graduate School of Engineering of Mie University. His current research interests are theoretical computer science and software. During 1985-1986, he worked at Passau University, F.R.G. as a research fellow of the AvH Foundation.

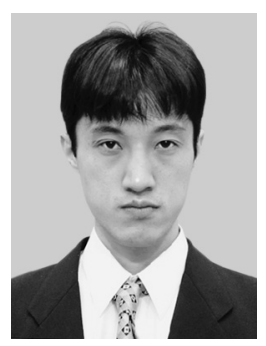

Kunihiro Matsuura was born in 1970 . He received the Ms. Eng. degree from Mie University in 1993. He is now a student of the Graduate School of Engineering of Mie University. His current research interest is term rewriting systems. 